University of Wollongong

Research Online

Faculty of Engineering and Information

Faculty of Engineering and Information

Sciences - Papers: Part B

Sciences

2019

Numerically pricing convertible bonds under stochastic volatility or stochastic interest rate with an ADI-based predictor-corrector scheme

Sha Lin

University of Wollongong, s1945@uowmail.edu.au

Song-Ping Zhu

University of Wollongong, spz@uow.edu.au

Follow this and additional works at: https://ro.uow.edu.au/eispapers1

Part of the Engineering Commons, and the Science and Technology Studies Commons

Research Online is the open access institutional repository for the University of Wollongong. For further information contact the UOW Library: research-pubs@uow.edu.au 


\title{
Numerically pricing convertible bonds under stochastic volatility or stochastic interest rate with an ADI-based predictor-corrector scheme
}

\author{
Abstract \\ 2019 Elsevier Ltd In this paper, the pricing problem for the American-style convertible bonds with the \\ Heston stochastic volatility and that with the Cox-Ingersoll-Ross (CIR) stochastic interest rate are both \\ considered. Due to the complexity of both problems, resulting from an additional stochastic factor, it is \\ almost impossible to find any analytical solution. Therefore, a predictor-corrector scheme is chosen as \\ the numerical scheme to solve the partial differential equations (PDEs), with the Douglas-Rachford (D-R) \\ method being utilized as one of the Alternating Direction Implicit (ADI) methods for the correction step to \\ obtain the numerical solution. Finally, the accuracy of our approach is numerically verified, and different \\ properties of convertible bond price and the optimal conversion price are also demonstrated and \\ discussed through examples.

\section{Disciplines} \\ Engineering | Science and Technology Studies

\section{Publication Details} \\ Lin, S. \& Zhu, S. (2019). Numerically pricing convertible bonds under stochastic volatility or stochastic \\ interest rate with an ADI-based predictor-corrector scheme. Computers and Mathematics with \\ Applications,
}




\title{
Numerically pricing convertible bonds under stochastic volatility or stochastic interest rate with an ADI-based predictor-corrector scheme
}

\author{
Sha Lin * $\quad$ Song-Ping Zhu $†$
}

\begin{abstract}
In this paper, the pricing problem for the American-style convertible bonds with the Heston stochastic volatility and that with the Cox-Ingersoll-Ross (CIR) stochastic interest rate are both considered. Due to the complexity of both problems, resulting from an additional stochastic factor, it is almost impossible to find any analytical solution. Therefore, a predictor-corrector scheme is chosen as the numerical scheme to solve the partial differential equations (PDEs), with the Douglas-Rachford (D-R) method being utilized as one of the Alternating Direction Implicit (ADI) methods for the correction step to obtain the numerical solution. Finally, the accuracy of our approach is numerically verified, and different properties of convertible bond price and the optimal conversion price are also demonstrated and discussed through examples.
\end{abstract}

Keywords. American-style convertible bonds, Predictor-correct scheme, ADI method

*Corresponding author: School of Finance, Zhejiang Gongshang University, Hangzhou 310018, China.

${ }^{\dagger}$ School of Mathematics and Applied Statistics, University of Wollongong NSW 2522, Australia.

$¥$ An adjunct professor of Tianjin University of Finance and Economics, China. 


\section{Introduction}

A bond is an instrument that can be treated as the issuer borrowing money from the holders for a pre-specified period. If a clause is added to the contract so that the holders can choose to convert the bond into a predetermined number of stocks or not, it then becomes a convertible bond (CB). While it provides a great incentives to a bond holder to invest in CBs rather than a conventional bond, this additional right of the holder indeed makes its pricing problem much more complicated, since the bond price and the optimal conversion price $^{1}$ should be determined simultaneously.

In 1973, Black \& Scholes [3] proposed to model the underlying price with a geometric Brownian motion (GBM) for the option pricing problem, and shortly after, Ingersoll [25] and Brennan \& Schwartz [4] considered the valuation problem of CBs with the firm value being taken as underlying variable following this particular Black-Scholes (B-S) model. This approach was improved by McConnel \& Schwartz [30] by replacing the firm value with the stock price as the underlying variable since the firm value can not be directly observed in real markets. Since then, various approaches have been proposed to price CBs. For example, Nyborg [32] obtained a closed-form solution under the B-S model for a simple convertible bond, which can only be converted at maturity, while Zhu [43] presented an analytical solution under the same model for a convertible bond, which can be converted at any time on or before maturity, using the homotopy analysis method.

If the issuer or the holder of a $\mathrm{CB}$ is entitled with some additional rights, different kinds of CBs will be formulated, such as callable CBs, puttable CBs, resettable CBs and so on, making the corresponding pricing problem even more complex. Thus, numerical methods must be adopted in most cases. For example, Tsiveriotis \& Fernandes [38] priced the cash-only CBs by applying the finite difference method on the coupled B-S equations. Ohtake et al. [33] presented the definitions of the call and reset clause, depending on which

\footnotetext{
${ }^{1}$ The optimal conversion price is referred to as the critical stock price beyond which the holder will choose to convert the bond into stocks.
} 
resettable CBs were considered by Kimura \& Shinohara [26] with the Monte Carlo method. Recently, Zhu et al. [45] derived an integral equation formulation for pricing the puttable convertible bond.

Of course, the B-S model is usually not adequate to model the underlying price, and one of the most popular approaches is to introduce additional random variables into the B-S model, which can mainly be divided into two categories, i.e., stochastic interest rate models and stochastic volatility models. Examples in the former category include the Merton model [31], CEV model [9, 12], Vasicek model [39], Dothan model [15], BrennanSchwartz model [5], CIR-VR model [10], GBM model [29] and CIR model [11], while the Heston model [20] and Hull-White model [21] are very popular among many others included in the latter category. Unfortunately, the additional random variables make the pricing problem much more complicated, and thus the numerical methods are often resorted to in these cases. In particular, the finite difference approach [42], the finite element approach [2], the finite volume approach [47], the binomial tree method [7, 23] and the Monte Carlo simulation method $[1,28]$ have already been adopted to price the convertible bonds under these complex models.

Another popular numerical approach is the predictor-corrector scheme [6, 35]. It is a method to solve the ordinary differential equation (ODE) with two steps; a prediction step computing the value of the function at a preceding set of points to obtain the value of this function at a subsequent point, and then a correction step refining the value of the unknown function at the same subsequent point using a suitable approach. In other words, it is a method with suitable association of an implicit scheme and an explicit scheme. In fact, this method has already been applied to solve the pricing problem of the security instruments, even though the governing equations for these pricing problems are all partial differential equations (PDEs). A typical example is provided in [46], where Zhu \& Zhang chose a suitable combination of a prediction scheme and a correction scheme to obtain a new scheme for evaluating American options under the B-S model. On the other hand, the 
Alternating Direction Implicit (ADI) method is a very useful technique to solve the PDEs on the rectangular domains $[13,14,22,34]$, especially for the parabolic ones as for the other two cases the problem can become quite complex [37]. Fortunately, the equations governing the prices of financial derivatives under most existing models, including the B-S model and the stochastic volatility/interest-rate models, are all parabolic differential equations, and thus the ADI method is ideal to be utilized for these pricing problems [18, 24].

In this paper, we adopt a particular predictor-corrector scheme, constructed by the two methods mentioned above being combined together with the ADI scheme used as the correction step. It was proposed by Zhu \& Chen [44] in solving the pricing problem of American puts with stochastic volatility, which was utilized by Chen et al [8] for the pricing of the stock loan with stochastic interest rate. In order to determine the price of CBs, we firstly establish two PDE systems for the price of CBs under a stochastic volatility and a stochastic interest rate model, respectively. Since the moving boundary exists in both of the two systems, Landau transform [27] is used to transform the free boundary problem into a fixed one, at the cost of the original linear PDE becoming a nonlinear one, after which the predictor-corrector scheme is adopted for each time step to convert the nonlinear PDE into two linearized difference equations associated with the prediction and correction phase, respectively. For the prediction step, an explicit Euler scheme is used to predict the value of the optimal conversion boundary, and at the correction step, the value of the bond is then determined through the ADI scheme, based on which the correction of the optimal conversion boundary is obtained. Another contribution of this paper is the proposition of the boundary conditions along the volatility and interest rate direction, which contribute to the development of the closed PDE system for pricing CBs under stochastic volatility and interest rate models.

The paper is organized as follows. In Section 2, the pricing problem for CBs under a stochastic volatility model is considered, presenting the numerical scheme as well as the numerical results we obtain. In Section 3, how to price CBs under a stochastic interest 
rate model is illustrated. Concluding remarks are given in the last section.

\section{Pricing convertible bonds with stochastic volatility}

In this section, the pricing problem of convertible bonds when the volatility is made to be another random variable is discussed. We will use the Heston model as an example to illustrate this since the processes in solving the pricing problem under different stochastic volatility models are very similar and the Heston model is one of the most popular models.

In the following, the PDE system governing the price of the CBs is firstly set up and then how to obtain the predictor-corrector scheme with the ADI method to value the CBs are illustrated, after which the accuracy of the proposed method is demonstrated through numerical experiments and the properties of the CBs with stochastic volatility are also studied.

\subsection{The PDE system under the Heston model}

To build the PDE system for pricing a CB, the dynamics of the adopted model should be specified first. Let $S_{t}$ be the underlying asset price, and then its dynamic under a riskneutral measure is assumed to satisfy the following stochastic different equation (SDE):

$$
d S_{t}=\left(r-D_{0}\right) S_{t} d t+\sqrt{v_{t}} S_{t} d W_{1}
$$

where $r$ is the risk-free interest rate and $D_{0}$ is the continuous dividend rate. $W_{1}$ is a standard Brownian motion, and $v_{t}$ is the stochastic volatility, which is governed by the following SDE:

$$
d v_{t}=\kappa\left(\eta-v_{t}\right) d t+\sigma \sqrt{v_{t}} d W_{2}
$$

with $\kappa$ denoting the rate of relaxation to this mean, $\eta$ representing the long time mean

of $v_{t}$, and $\sigma$ being the volatility of volatility. $W_{2}$ is also a standard Brownian motion, 
being correlated $W_{1}$ with $\rho \in[-1,1]$. It should be noted that as $v_{t}$ represents the value of volatility, it should be greater than 0, and thus the so-called Feller condition [16] should be imposed here to ensure that $v_{t}>0$ almost surely, which requires that the parameters of the volatility process satisfy $\kappa \eta>\frac{1}{2} \sigma^{2}$. If the value of the bond is denoted by $U(S, v, t)$, its governing PDE can be written as follows:

$$
\frac{1}{2} v S^{2} \frac{\partial^{2} U}{\partial S^{2}}+\sigma \rho v S \frac{\partial^{2} U}{\partial S \partial v}+\frac{1}{2} \sigma^{2} v \frac{\partial^{2} U}{\partial v^{2}}+\left(r-D_{0}\right) S \frac{\partial U}{\partial S}+\kappa(\eta-v) \frac{\partial U}{\partial v}-r U+\frac{\partial U}{\partial t}=0
$$

The terminal condition for Equation (2.3) is actually the payoff function of CBs

$$
U(S, v, T)=\max \left\{C_{R} S, Z\right\}
$$

where $C_{R}$ is the conversion ratio and $Z$ is the face value of the bond. According to the definition of a convertible bond, its holder can choose to convert it into a pre-determined number of stocks when the stock price is high enough, and thus the continuation region should be $S \in\left(0, S_{f}(v, t)\right]$, with $S_{f}(v, t)$ being the optimal conversion price. Therefore, boundary conditions should be imposed along $S=0$ and $S=S_{f}(v, t)$. When $S=0$, the holder would never choose conversion, and instead, the bond would be held until the expiry so that the holder could receive the face value. In this sense, taking into account the discounting factor, the value of a CB should satisfy

$$
\lim _{S \rightarrow 0} U(S, v, t)=Z e^{-r(T-t)}
$$

The boundary conditions at the optimal conversion boundary $S=S_{f}(v, t)$ can be derived using the no-arbitrage argument $[40]$

$$
\begin{aligned}
& U\left(S_{f}(v, t), v, t\right)=C_{R} \cdot S_{f}(v, t), \\
& \frac{\partial U}{\partial S}\left(S_{f}(v, t), v, t\right)=C_{R} .
\end{aligned}
$$


It should be noted that all of the conditions mentioned above are very similar to that under the B-S model, and the main difference between the B-S model and the stochastic volatility model is that the bond price and the optimal conversion price are both the functions of the volatility for the stochastic volatility model. Therefore, the boundary conditions for $v$ are needed to close the PDE system. In this study, the boundary conditions are chosen as

$$
\begin{aligned}
& \lim _{v \rightarrow 0} U(S, v, t)=\max \left\{C_{R} S, Z e^{-r(T-t)}\right\}, \\
& \lim _{v \rightarrow \infty} \frac{\partial U}{\partial v}(S, v, t)=0 .
\end{aligned}
$$

We would like to explain a bit on how we choose the boundary conditions in the direction of $v$. On one hand, for the boundary condition at $v=0$, it needs to be pointed out that this boundary condition is not necessary if the Fichera function [17] along $v=0$ satisfies $\kappa \eta-\frac{\sigma^{2}}{2} \geq 0$, while the boundary condition at $v=0$ is needed to close the system when $\kappa \eta<\frac{\sigma^{2}}{2}$. Clearly, when the Feller condition is imposed, no boundary condition along $v=0$ is needed as the system is already closed. However, if one only analyzes PDE (2.3), values of $\kappa, \eta$ and $\sigma$ can of course be selected such that $\kappa \eta<\frac{\sigma^{2}}{2}$, in which case a certain boundary condition is still needed to ensure the uniqueness of the solution. Therefore, financial arguments will be used to derive an appropriate boundary condition for this case. The solution of $\operatorname{SDE}(2.1)$ when $v=0$ can be approximated as, $S=e^{\left(r-D_{0}\right) t} S_{0}$, there is virtually no risk with the underlying asset. This demonstrates that if $C_{R} S>Z e^{-r(T-t)}$, there is no sense to hold the bond, and it should be exercised immediately, implying that the bond price at this situation is $C_{R} S$. In contrast, if $C_{R} S \leq Z e^{-r(T-t)}$, the bond should be held until the expiry and its value should be $Z e^{-r(T-t)}$ instead. Therefore, both cases show that the boundary condition at $v=0$ is $\lim _{v \rightarrow 0} U(S, v, t)=\max \left\{C_{R} S, Z e^{-r(T-t)}\right\}$. On the other hand, when the volatility approaches infinity, the bond price should be independent of the volatility change, otherwise, the bond price will reach infinity, since the bond price 
is an increasing function with respect to the volatility.

Combining all the discussions above, the PDE system for pricing a CB under the Heston model can be summarized as

$$
\left\{\begin{array}{l}
\frac{1}{2} v S^{2} \frac{\partial^{2} U}{\partial S^{2}}+\sigma \rho v S \frac{\partial^{2} U}{\partial S \partial v}+\frac{1}{2} \sigma^{2} v \frac{\partial^{2} U}{\partial v^{2}}+\left(r-D_{0}\right) S \frac{\partial U}{\partial S} \\
\quad+\kappa(\eta-v) \frac{\partial U}{\partial v}-r U+\frac{\partial U}{\partial t}=0 \\
U(S, v, T)=\max \left\{C_{R} S, Z\right\}, \\
\lim _{S \rightarrow 0} U(S, v, t)=Z e^{-r(T-t)}, \\
U\left(S_{f}(v, t), v, t\right)=C_{R} \cdot S_{f}(v, t), \\
\frac{\partial U}{\partial S}\left(S_{f}(v, t), v, t\right)=C_{R}, \\
\lim _{v \rightarrow 0} U(S, v, t)=\max \left\{C_{R} S, Z e^{-r(T-t)}\right\}, \\
\lim _{v \rightarrow \infty} \frac{\partial U}{\partial v}(S, v, t)=0,
\end{array}\right.
$$

for $S \in\left(0, S_{f}(v, t)\right], v \in(0, \infty)$ and $t \in[0, T]$. In the following, we are going to present the details on how to apply the predictor-corrector method with ADI scheme on the PDE system governing the value of CBs.

It should be remarked that although CBs considered in this paper and American put options discussed in [44] are both American-style financial derivatives, the corresponding pricing PDE systems are quite different because of the nature of the two contracts. The main difference that distinguishes the two PDE systems is the domain of the underlying price; our PDE system is restricted on a finite domain, $S \in\left(0, S_{f}(v, t)\right]$, as CBs are similar to American call options, while the PDE system presented in [44] holds on a semi-infinite domain, $S \in\left(S_{f}(v, t), \infty\right)$.

\subsection{Discretize the PDE system}

In this subsection, the PDE system is discretized with some rules. Before discretization, it should be noted that one of the boundaries of System (2.10) in the direction of $S$ is 
not fixed, which poses an obstacle in applying the predictor-corrector method. Therefore, a classical transform, Landau transform [27], i.e. $x=\ln \left(\frac{S}{S_{f}}\right)$, should be adopted to this PDE system to solve this issue, and at the same time the value of the optimal conversion boundary is now a part of the solution. Moreover, a simple transform, $\tau=T-t$, is also applied to the PDE system to change the terminal condition problem to an initial counterpart. In addition, another transform, $V(x, v, \tau)=U(x, v, \tau)-Z e^{-r \tau}$, is also made here so that the PDE system (2.10) can be rewritten as

$$
\left\{\begin{array}{l}
\mathcal{L} V(x, v, \tau)=0, \\
V(x, v, 0)=\max \left\{C_{R} \cdot S_{f}(v, 0) \cdot e^{x}-Z, 0\right\} \\
\lim _{x \rightarrow-\infty} V(x, v, \tau)=0, \\
V(0, v, \tau)=C_{R} \cdot S_{f}(v, \tau)-Z e^{-r \tau}, \\
\frac{\partial V}{\partial x}(0, v, \tau)=C_{R} \cdot S_{f}(v, \tau), \\
\lim _{v \rightarrow 0} V(x, v, \tau)=\max \left\{C_{R} \cdot S_{f}(0, \tau) \cdot e^{x}-Z e^{-r \tau}, 0\right\} \\
\lim _{v \rightarrow \infty} \frac{\partial V}{\partial v}(x, v, \tau)=0,
\end{array}\right.
$$

with $x \in(-\infty, 0], v \in(0, \infty)$ and $\tau \in[0, T]$, and

$$
\begin{aligned}
\mathcal{L}= & {\left[\frac{1}{2} v+\frac{1}{2} \sigma^{2} v \frac{1}{S_{f}^{2}}\left(\frac{\partial S_{f}}{\partial v}\right)^{2}-\frac{\rho \sigma v}{S_{f}} \frac{\partial S_{f}}{\partial v}\right] \frac{\partial^{2}}{\partial x^{2}}+\frac{1}{2} \sigma^{2} v \frac{\partial^{2}}{\partial v^{2}}+\left(\rho \sigma v-\frac{\sigma^{2} v}{S_{f}} \frac{\partial S_{f}}{\partial v}\right) \frac{\partial^{2}}{\partial x \partial v} } \\
& +\left[-\frac{1}{2} v+\frac{1}{2} \frac{\sigma^{2} v}{S_{f}^{2}}\left(\frac{\partial S_{f}}{\partial v}\right)^{2}-\frac{1}{2} \frac{\sigma^{2} v}{S_{f}} \frac{\partial^{2} S_{f}}{\partial v^{2}}+r-D_{0}-\kappa(\eta-v) \frac{1}{S_{f}} \frac{\partial S_{f}}{\partial v}+\frac{1}{S_{f}} \frac{\partial S_{f}}{\partial \tau}\right] \frac{\partial}{\partial \tau} \\
& +\kappa(\eta-v) \frac{\partial}{\partial v}-r-\frac{\partial}{\partial \tau} .
\end{aligned}
$$

To further simplify the notation of $\mathcal{L}$, some new notations are defined here

$$
\xi=\frac{1}{S_{f}} \cdot \frac{\partial S_{f}}{\partial v}, \quad \beta=\frac{1}{S_{f}} \cdot \frac{\partial^{2} S_{f}}{\partial v^{2}}, \quad \lambda=\frac{1}{S_{f}} \cdot \frac{\partial S_{f}}{\partial \tau}
$$


with which $\mathcal{L}$ can be represented by

$$
\mathcal{L}=a(v) \frac{\partial^{2}}{\partial x^{2}}+b(v) \frac{\partial^{2}}{\partial v^{2}}+c(v) \frac{\partial^{2}}{\partial x \partial v}+[d(v)+\lambda] \frac{\partial}{\partial x}+e(v) \frac{\partial}{\partial v}-r-\frac{\partial}{\partial \tau}
$$

where

$$
\begin{aligned}
a(v) & =\frac{1}{2} v+\frac{1}{2} \sigma^{2} v \xi^{2}-\rho \sigma v \xi, \\
b(v) & =\frac{1}{2} \sigma^{2} v, \\
c(v) & =\rho \sigma v-\sigma^{2} v \xi \\
d(v) & =-\frac{1}{2} v+\frac{1}{2} \xi^{2} \sigma^{2} v-\frac{1}{2} \sigma^{2} v \beta+r-D_{0}-\kappa(\eta-v) \xi, \\
e(v) & =\kappa(\eta-v) .
\end{aligned}
$$

Before our approach can be applied to obtain the numerical solution, System (2.11) should be discretized first. Specifically, the semi-infinite domain should be firstly truncated into a finite one as follows

$$
\left\{(x, v, \tau) \mid x \in\left[x_{\min }, 0\right], v \in\left[0, v_{\max }\right], \tau \in[0, T]\right\}
$$

and the values of $x_{\min }$ and $v_{\max }$ will be chosen when we present the numerical results. Then, the finite domain will be separated into $N_{\tau}$ uniform grids in the direction of $\tau, N_{x}$ uniform grids in the direction of $x$ and $N_{v}$ uniform grids in the direction of $v$. Thus, we have

$$
\Delta \tau=\frac{T}{N_{\tau}}, \quad \Delta x=-\frac{x_{\min }}{N_{x}}, \quad \Delta v=\frac{v_{\max }}{N_{v}}
$$

and

$$
\begin{aligned}
& \tau_{n}=n \Delta \tau, \quad \text { with } \tau_{0}=0 \text { and } \tau_{N_{\tau}}=T, \\
& x_{i}=x_{\min }+i \Delta x, \text { with } x_{0}=x_{\min } \text { and } x_{N_{x}}=0,
\end{aligned}
$$




$$
v_{j}=j \Delta v, \quad \text { with } v_{0}=0 \text { and } v_{N_{v}}=v_{\max } .
$$

In this case, the value of the unknown functions at a grid point, $V\left(x_{i}, v_{j}, \tau_{n}\right)$ and $S_{f}\left(v_{j}, \tau_{n}\right)$, are denoted as $V_{i, j}^{(n)}$ and $S_{f}^{(n)}(j)$, respectively, for $i=0,1, \cdots, N_{x}, j=0,1, \cdots, N_{v}$ and $n=0,1, \cdots, N_{\tau}$.

In order to apply our numerical method, we now classify the entire domain into two parts, with the first one being the interior of the domain

$$
\mathcal{D}=\left\{\left(x_{i}, v_{j}\right) \mid i=1, \cdots, N_{x}-1, j=1, \cdots, N_{v}-1\right\},
$$

and another one representing the boundaries. For each grid point in $\mathcal{D}$, the standard central difference scheme and the second-order half-central difference scheme are used to approximate the first-order derivative (including the cross-derivative) and the second-order derivative, respectively. Thus, all the derivatives belonging to $\mathcal{D}$ are discretized as following:

$$
\begin{aligned}
\frac{\partial V_{i, j}^{(n)}}{\partial x} & =\frac{V_{i+1, j}^{(n)}-V_{i-1, j}^{(n)}}{2 \Delta x}, \\
\frac{\partial V_{i, j}^{(n)}}{\partial v} & =\frac{V_{i, j+1}^{(n)}-V_{i, j-1}^{(n)}}{2 \Delta v}, \\
\frac{\partial^{2} V_{i, j}^{(n)}}{\partial x^{2}} & =\frac{V_{i+1, j}^{(n)}-2 V_{i, j}^{(n)}+V_{i-1, j}^{(n)}}{(\Delta x)^{2}} \\
\frac{\partial^{2} V_{i, j}^{(n)}}{\partial v^{2}} & =\frac{V_{i, j+1}^{(n)}-2 V_{i, j}^{(n)}+V_{i, j-1}^{(n)}}{(\Delta v)^{2}}, \\
\frac{\partial^{2} V_{i, j}^{(n)}}{\partial x \partial v} & =\frac{V_{i+1, j+1}^{(n)}-V_{i+1, j-1}^{(n)}-V_{i-1, j+1}^{(n)}+V_{i-1, j-1}^{(n)}}{4 \Delta v \Delta x} .
\end{aligned}
$$

For the boundary part, it is easy to deal with the Dirichlet boundary conditions, while it is quite difficult to approximate the Neumann boundary condition. This is because in general, the first-order derivative at $x=0$ should be presented

$$
\frac{\partial V_{N_{x}, j}^{(n)}}{\partial x}=\frac{V_{N_{x}+1, j}^{(n)}-V_{N_{x}-1, j}^{(n)}}{2 \Delta x}
$$


whereas it is impossible to obtain the value of $V_{N_{x}+1, j}^{(n)}$. Therefore, we have to use an alternative approach, the so-called one-sided difference, instead of the central one. It is a form of extrapolation that determines the value of the unknown function on the boundary in terms of its values at the interior grid points [37]. With the use of the Taylor series, we can obtain the following equations:

$$
\begin{aligned}
V_{N_{x}-1}^{(n)} & =V_{N_{x}, j}^{(n)}-\Delta x \frac{\partial V_{N_{x}, j}^{(n)}}{\partial x}+\frac{1}{2}(\Delta x)^{2} \frac{\partial^{2} V_{N_{x}, j}^{(n)}}{\partial x^{2}}+o\left((\Delta x)^{3}\right), \\
V_{N_{x}-2, j}^{(n)} & =V_{N_{x}, j}^{(n)}-2 \Delta x \frac{\partial V_{N_{x}, j}^{(n)}}{\partial x}+\frac{1}{2}(2 \Delta x)^{2} \frac{\partial^{2} V_{N_{x}, j}^{(n)}}{\partial x^{2}}+o\left((\Delta x)^{3}\right) .
\end{aligned}
$$

By eliminating $\frac{\partial^{2} V_{N_{x}, j}^{(n)}}{\partial x^{2}}$, we can further obtain

$$
\frac{\partial V_{N_{x}, j}^{(n)}}{\partial x}=\frac{3 V_{N_{x}, j}^{(n)}+V_{N_{x}-2, j}^{(n)}-4 V_{N_{x}-1, j}^{(n)}}{2 \Delta x}+o\left((\Delta x)^{3}\right)
$$

where the value of $\frac{\partial V_{N_{x}, j}^{(n)}}{\partial x}$ is expressed in the form of the values for $V_{N_{x}-2, j}^{(n)}, V_{N_{x}-1, j}^{(n)}$, and the unknown boundary value $V_{N_{x}, j}^{(n)}$ approximately. In summary, the finite difference equation (FDE) system written on a grid point for System (2.11) can be specified as

$$
\left\{\begin{array}{l}
\frac{\partial V_{i, j}^{(n)}}{\partial \tau}=a_{j} \frac{\partial^{2} V_{i, j}^{(n)}}{\partial x^{2}}+b_{j} \frac{\partial^{2} V_{i, j}^{(n)}}{\partial v^{2}}+c_{j} \frac{\partial^{2} V_{i, j}^{(n)}}{\partial x \partial v}+\left[d_{j}+\lambda_{j}\right] \frac{\partial V_{i, j}^{(n)}}{\partial x}+e_{j} \frac{\partial V_{i, j}^{(n)}}{\partial v}-r V_{i, j}^{(n)}, \\
V_{i, j}^{(0)}=\max \left\{C_{R} \cdot S_{f}^{(0)}(j) e^{x_{i}}-Z, 0\right\}, \\
V_{0, j}^{(n)}=0 \\
V_{N_{x}, j}^{(n)}=C_{R} \cdot S_{f}^{(n)}(j)-Z e^{-r \tau_{n}}, \\
\frac{3 V_{N_{x}, j}^{(n)}+V_{N_{x}-2, j}^{(n)}-4 V_{N_{x}-1, j}^{(n)}}{2 \Delta x}=C_{R} \cdot S_{f}^{(n)}(j), \\
V_{i, 0}^{(n)}=\max \left\{C_{R} \cdot S_{f}^{(n)}(0) e^{x_{i}}-Z e^{-r \tau_{n}}, 0\right\}, \\
V_{i, N_{v}}^{(n)}=0
\end{array}\right.
$$

where

$$
a_{j}=\frac{1}{2} v_{j}+\frac{1}{2} \sigma^{2} v_{j} \xi_{j}^{2}-\rho \sigma v_{j} \xi_{j}
$$




$$
\begin{aligned}
b_{j} & =\frac{1}{2} \sigma^{2} v_{j} \\
c_{j} & =\rho \sigma v_{j}-\sigma^{2} v_{j} \xi_{j} \\
d_{j} & =-\frac{1}{2} v_{j}+\frac{1}{2} \sigma^{2} v_{j} \xi_{j}-\frac{1}{2} \sigma^{2} v_{j} \beta_{j}+r-D_{0}-\kappa\left(\eta-v_{j}\right) \xi_{j}, \\
e_{j} & =\kappa\left(\eta-v_{j}\right)
\end{aligned}
$$

with

$$
\begin{aligned}
\xi_{j} & =\frac{1}{S_{f}(j)} \cdot \frac{S_{f}(j+1)-S_{f}(j-1)}{2 \Delta v}, \\
\beta_{j} & =\frac{1}{S_{f}(j)} \cdot \frac{S_{f}(j+1)-2 S_{f}(j)+S_{f}(j-1)}{(\Delta v)^{2}}, \\
\lambda_{j} & =\frac{1}{S_{f}(j)} \cdot \frac{\partial S_{f}(j)}{\partial \tau} .
\end{aligned}
$$

It should be remarked here that the boundary condition in the FDE system is changed from the Neumann one to the Dirichlet counterpart when $v \rightarrow \infty$. This can be explained by nothing that the initial Neumann boundary condition implies that the value of the bond at $v \rightarrow \infty$ should be a constant (independent on $v$ ), and such a constant should be equal to 0 since the holder will not choose to convert the bond as the market is too volatile and the bond price in this case should equal to $Z e^{-r \tau}$, or in other words, $\lim _{v \rightarrow \infty} V(x, v, \tau)=0$. Another thing should also be noted that the time derivatives have not been discretized by now, and the explicit Euler scheme and the implicit Euler scheme are applied to the time derivative $\frac{\partial V_{i, j}^{(n)}}{\partial \tau}$ and $\frac{\partial S_{f}^{(n)}(j)}{\partial \tau}$, respectively, in the process of applying the predictorcorrector scheme, the details of which are illustrated in the next two subsections.

\subsection{Numerical scheme for the prediction step}

It should be pointed out that the PDE system (2.35) is a linear one if the values of the optimal conversion price $S_{f}^{(n)}$ are known at the beginning of the $(n+1)$ th step, in which case the bond price at the $(n+1)$ th step can be directly derived. Thus, the prediction step 
that is used to predict the value of the optimal conversion boundary is actually a process of linearizing the nonlinear PDE system.

Firstly, we should recall the discretized formulation of the boundary conditions at $x=0$

$$
\begin{aligned}
V_{N_{x}, j}^{(n)} & =C_{R} \cdot S_{f}^{(n)}(j)-Z e^{-r \tau_{n}} \\
C_{R} \cdot S_{f}^{(n)}(j) & =\frac{3 V_{N_{x}, j}^{(n)}+V_{N_{x}-2, j}^{(n)}-4 V_{N_{x}-1, j}^{(n)}}{2 \Delta x} .
\end{aligned}
$$

Then, we can obtain

$$
\begin{aligned}
& C_{R} \cdot S_{f}^{(n)}(j)=\frac{3 V_{N_{x}, j}^{(n)}+V_{N_{x}-2, j}^{(n)}-4 V_{N_{x}-1, j}^{(n)}}{2 \Delta x} \\
\Rightarrow & C_{R} \cdot S_{f}^{(n)}(j)=\frac{3\left(C_{R} \cdot S_{f}^{(n)}(j)-Z e^{-r \tau_{n}}\right)+V_{N_{x}-2, j}^{(n)}-4 V_{N_{x}-1, j}^{(n)}}{2 \Delta x} \\
\Rightarrow & 2 \Delta x \cdot C_{R} \cdot S_{f}^{(n)}(j)=3\left(C_{R} \cdot S_{f}^{(n)}(j)-Z e^{-r \tau_{n}}\right)+V_{N_{x}-2, j}^{(n)}-4 V_{N_{x}-1, j}^{(n)} \\
\Rightarrow & (3-2 \Delta x) \cdot C_{R} \cdot S_{f}^{(n)}(j)=3 Z e^{-r \tau_{n}}+4 V_{N_{x}-1, j}^{(n)}-V_{N_{x}-2, j}^{(n)} \\
\Rightarrow & S_{f}^{(n)}(j)=\frac{3 Z e^{-r \tau_{n}}+4 V_{N_{x}-1, j}^{(n)}-V_{N_{x}-2, j}^{(n)}}{(3-2 \Delta x) \cdot C_{R}} .
\end{aligned}
$$

If we assume the values of the bond and its optimal conversion boundary at the $n$th time step are known, the optimal conversion boundary at the $(n+1)$ th time step can be determined by using the formulation above

$$
S_{f}^{(n+1)}(j)=\frac{3 Z e^{-r \tau_{n+1}}+4 V_{N_{x}-1, j}^{(n+1)}-V_{N_{x}-2, j}^{(n+1)}}{(3-2 \Delta x) \cdot C_{R}}
$$

With this newly obtained expression, if the explicit Euler scheme and the implicit Euler scheme are applied to the time derivative $\frac{\partial V_{i, j}^{(n)}}{\partial \tau}$ and $\frac{\partial S_{f}^{(n)}(j)}{\partial \tau}$, respectively, we can obtain

$$
\begin{aligned}
V_{i, j}^{(n+1)}=V_{i, j}^{(n)}+\Delta & \left\{\left\{a_{j} \frac{\partial^{2} V_{i, j}^{(n)}}{\partial x^{2}}+b_{j} \frac{\partial^{2} V_{i, j}^{(n)}}{\partial v^{2}}+c_{j} \frac{\partial^{2} V_{i, j}^{(n)}}{\partial x \partial v}+\left[d_{j}+\frac{S_{f}^{(n+1)}(j)-S_{f}^{(n)}(j)}{\Delta \tau S_{f}^{(n)}(j)}\right] \frac{\partial V_{i, j}^{(n)}}{\partial x}\right.\right. \\
+ & \left.e_{j} \frac{\partial V_{i, j}^{(n)}}{\partial v}\right\}-r \Delta \tau V_{i, j}^{(n)}, \quad i=N_{x}-2, N_{x}-1 .
\end{aligned}
$$


After some complex computation, the predicted value of the optimal conversion boundary at $(n+1)$ th time step, ${\tilde{S_{f}}}^{(n+1)}(j)$, can be presented

$$
{\tilde{S_{f}}}^{(n+1)}(j)=\frac{3 Z e^{-r \tau_{n+1}}+\mathcal{F}\left[4 V_{N_{x}-1, j}^{(n)}-V_{N_{x}-2, j}^{(n)}\right]}{(3-2 \Delta x) C_{R}-\frac{\delta_{x}\left(4 V_{N_{x}-1, j}^{(n)}-V_{N_{x}-2, j}^{(n)}\right)}{S_{f}^{(n)}(j)}},
$$

with

$$
\mathcal{F}=\mathbb{I}+\Delta \tau\left[a_{j} \delta_{x x}+b_{j} \delta_{v v}+c_{j} \delta_{x v}+\left(d_{j}-\frac{1}{\Delta \tau}\right) \delta_{x}+e_{j} \delta_{v}\right]-r \Delta \tau
$$

Here, all the derivatives are replaced by the corresponding $\delta_{*}$. Therefore, the predicted value of the corresponding bond price, $\tilde{V}_{N_{x}, j}^{(n+1)}$, can be calculated as

$$
\tilde{V}_{N_{x}, j}^{(n+1)}=C_{R} \cdot{\tilde{S_{f}}}^{(n+1)}(j)-Z e^{-r \tau_{n+1}}
$$

In summary, the value of the optimal conversion boundary and the bond price on this boundary can be predicted by Equation (2.50) and Equation (2.51), respectively, with which the algorithm of ADI method is used to obtain all the values at the $(n+1)$ th time step, $V^{(n+1)}$, in the next subsection. Afterwards, we can correct the values of $S_{f}^{(n+1)}$ and $V_{N_{x}}^{(n+1)}$ by using the newly obtained $V_{N_{x}-2}^{(n+1)}$ and $V_{N_{x}-1}^{(n+1)}$.

\subsection{Numerical scheme for the correction step}

In this subsection, ADI method is utilized for the correction step. As mentioned above, this method is a very useful technique to solve PDEs on rectangular domains, especially for the parabolic ones. Moreover, what we choose in this study is the Douglas-Rachford (D-R) method, whose accuracy is of first-order in time and second-order in space. Now, the FDE should be rewritten, so that the ADI method can be applied

$$
\left(\mathbb{I}-\phi A_{1}\right)\left(\mathbb{I}-\phi A_{2}\right) V^{(n+1)}=\left[\mathbb{I}+A_{0}+(1-\phi) A_{1}+A_{2}\right] V^{(n)}-\left(\mathbb{I}-\phi A_{1}\right) \phi A_{2} V^{(n)},
$$


where $\phi \in[0,1]$. The procedures to obtain all operators, $A_{0}, A_{1}$ and $A_{2}$, are left in Appendix A. For the D-R method, there are two steps that need to be conducted before we can obtain the final scheme. Firstly, we should calculate the intermediate value, $Y$, with the following equation

$$
\left(\mathbb{I}-\phi A_{1}\right) Y=\left[\mathbb{I}+A_{0}+(1-\phi) A_{1}+A_{2}\right] V^{(n)},
$$

where we fix the $v$ direction. Then, the above equation can be simplified as

$$
\mathbb{A} Y_{j}=P_{j}+\mathbb{B} x_{j}
$$

with the details of $\mathbb{A}, Y_{j}, P_{j}$ and $\mathbb{B} x_{j}$ being presented in Appendix B. It should be noted that matrix $\mathbb{A}$ is a tridiagonal, and thus the Thomas algorithm [37] can be applied to improve the speed and the accuracy of our method. It should also be pointed out that the value $Y$ is actually obtained by the loop of $j$ and each $Y_{j}$ is a vector with $\left(N_{x}+1\right) \times 1$. Once the value of $Y$ is known, we can then compute $V^{(n+1)}$ from the following equation

$$
\left(\mathbb{I}-\phi A_{2}\right) V^{(n+1)}+\phi A_{2} V^{(n)}=Y
$$

with the $x$ direction being fixed. This equation can be further represented as

$$
\mathbb{C} V_{i}^{(n+1)}=Q_{i}+\mathbb{B} v_{i}
$$

and the details of $\mathbb{C}, V_{i}^{(n+1)}, Q_{i}$ and $\mathbb{B} v_{i}$ are left in Appendix C. Similarly to Equation (2.54), Matrix $\mathbb{C}$ is also a tridiagonal, and the Thomas algorithm is utilized here again. For this equation, the loop of $i$ is used to obtain the value of $V^{(n+1)}$, leading to our desired result, since it can be easily proved that solving Equation (2.54) and Equation (2.56) means solving the initial Equation (2.52). Clearly, with these two steps illustrated in this subsection, the algorithm to derive the corrector scheme has already been designed. 
In order to numerically implement our scheme in the next subsection, we need to make it clear how to calculate the boundary value of the intermediate value $Y$, which is presented as

$$
\begin{aligned}
Y_{0} & =0, \\
Y_{N_{x}} & =\left(\mathbb{I}-\phi A_{2}\right) \tilde{V}_{N_{x}}^{(n+1)}+\phi A_{2} V_{N_{x}}^{(n)} \\
& =\left(\mathbb{I}-\phi A_{2}\right)\left(C_{R} \cdot \tilde{S}_{f}^{(n+1)}-Z e^{-r \tau_{n+1}}\right)+\phi A_{2} V_{N_{x}}^{(n)},
\end{aligned}
$$

with the use of the predicted value of ${\tilde{S_{f}}}^{(n+1)}$. Clearly, this has completed the establishment of the predictor-corrector scheme with the ADI method for pricing CBs if we combine the predictor scheme presented in the previous subsection and the corrector scheme shown in this subsection.

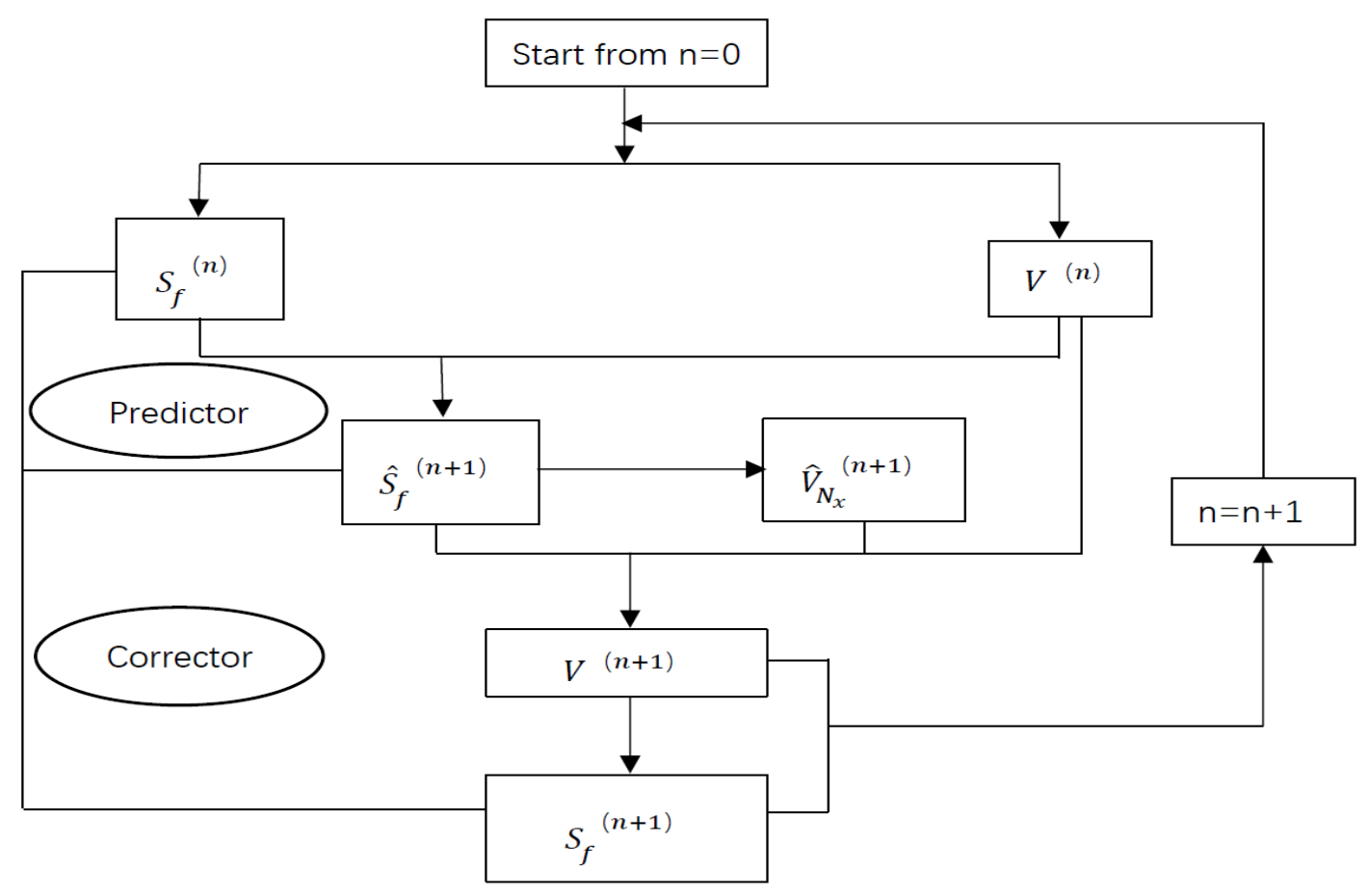

Figure 1: Schematic flow chart of the scheme.

To make it easier for readers to understand this approach, a schematic flow chart on how this scheme can be implemented is shown in Figure 1. Assuming that the values of 
$S_{f}^{(n)}$ and $V^{(n)}$ have been obtained from the last loop, the values of $\tilde{S}_{f}^{(n+1)}$ and $\tilde{V}_{N_{x}}^{(n+1)}$ can then be derived utilizing Equations (2.49) and (2.51), respectively, in the predictor phase. Based on these, the value of $V^{n+1}$ can be obtained using the ADI scheme, which also gives

rise to that of $S_{f}^{(n+1)}$, in the corrector phase. By repeating this process until the expiry time, we are able to calculate all the values for the target bond prices as well as optimal conversion boundary. Following this, we are now ready to conduct numerical experiments to study the properties of CBs under the stochastic volatility model, the details of which are shown in the next subsection.

Before we present any numerical results, it should be pointed out that the discussion on the convergence of the scheme is omitted here as the PDE considered here is the same as the one discussed in [44]. Interested readers are referred to [44] or Section 3.2 of this paper for a similar discussion.

\subsection{Numerical examples}

In this subsection, the accuracy of our method is tested first, and then the numerical results are provided to illustrate several properties of the convertible bond under a stochastic volatility model. Unless otherwise stated, the parameters used are listed below:

- Face value $Z=10$,

- Conversion ratio $C_{R}=1$,

- Maturity $T=1$ (year),

- Risk-free annual interest rate $r=0.1$,

- Rate of continuous dividend payment $D_{0}=0.07$,

- Reversion rate $\kappa=1.5$,

- Reversion level $\eta=0.16$, 
- Volatility of the volatility $\sigma=0.4$,

- Correlation factor $\rho=0.1$.

Before we present the numerical results, it is necessary to determine the domain that we are going to operate on. Although $x$ can take any value being less than 0 , we need to truncate the semi-infinite domain into a finite one so as to implement our numerical scheme. As mentioned in [40] that it suffices to set the minimum of $x$ to be $-\ln 5$, the domain of our model is assumed as

$$
\{(x, v, \tau) \mid x \in[-\ln 5,0] \times[0,1] \times[0,1]\}
$$

Here, setting $v_{\max }=1$ is sufficient and reasonable since the value of the volatility is usually very small. After the domain is uniformly discretized, with the step size in the direction of $x, v$ and $t$ being 101, 201 and 5001, respectively, the numerical results are presented in the following. It should also be pointed out that all of our calculations in this paper are done using Matlab R2017a on a PC with the following specifications: Intel(R) Core(TM), i7-4790 CPU@3.60GHz 3.60 GHz, and 16.0 GB of RAM.

Table 1: Convergence test on the optimal conversion boundary

\begin{tabular}{c|c|c|c|c}
\hline Volatility value & $(N x, N v, N t)$ & ADI & IE & Relative error \\
\hline \multirow{4}{*}{$v=0.1$} & $(25,50,1250)$ & 12.8669 & & $5.97 \times 10^{-3}$ \\
& $(50,100,2500)$ & 12.8110 & 12.7905 & $1.61 \times 10^{-3}$ \\
& $(100,200,5000)$ & 12.7969 & & $5.00 \times 10^{-4}$ \\
\hline \multirow{3}{*}{$v=0.2$} & $(25,50,1250)$ & 15.7500 & & $6.17 \times 10^{-3}$ \\
& $(50,100,2500)$ & 15.6796 & 15.6533 & $1.67 \times 10^{-3}$ \\
& $(100,200,5000)$ & 15.6618 & & $5.38 \times 10^{-4}$ \\
\hline \multirow{3}{*}{$v=0.4$} & $(25,50,1250)$ & 21.2244 & & $8.19 \times 10^{-3}$ \\
& $(50,100,2500)$ & 21.1017 & 21.0519 & $2.36 \times 10^{-3}$ \\
& $(100,200,5000)$ & 21.0709 & & $9.00 \times 10^{-4}$ \\
\hline
\end{tabular}

To validate our numerical scheme, a degenerate case is considered as the benchmark, where the volatility is a fixed value instead of being stochastic, and the values of the optimal conversion boundary calculated with our method and those derived through the integral equation approach [45] are displayed in both Table 1 and Figure 2. In particular, Table 1 


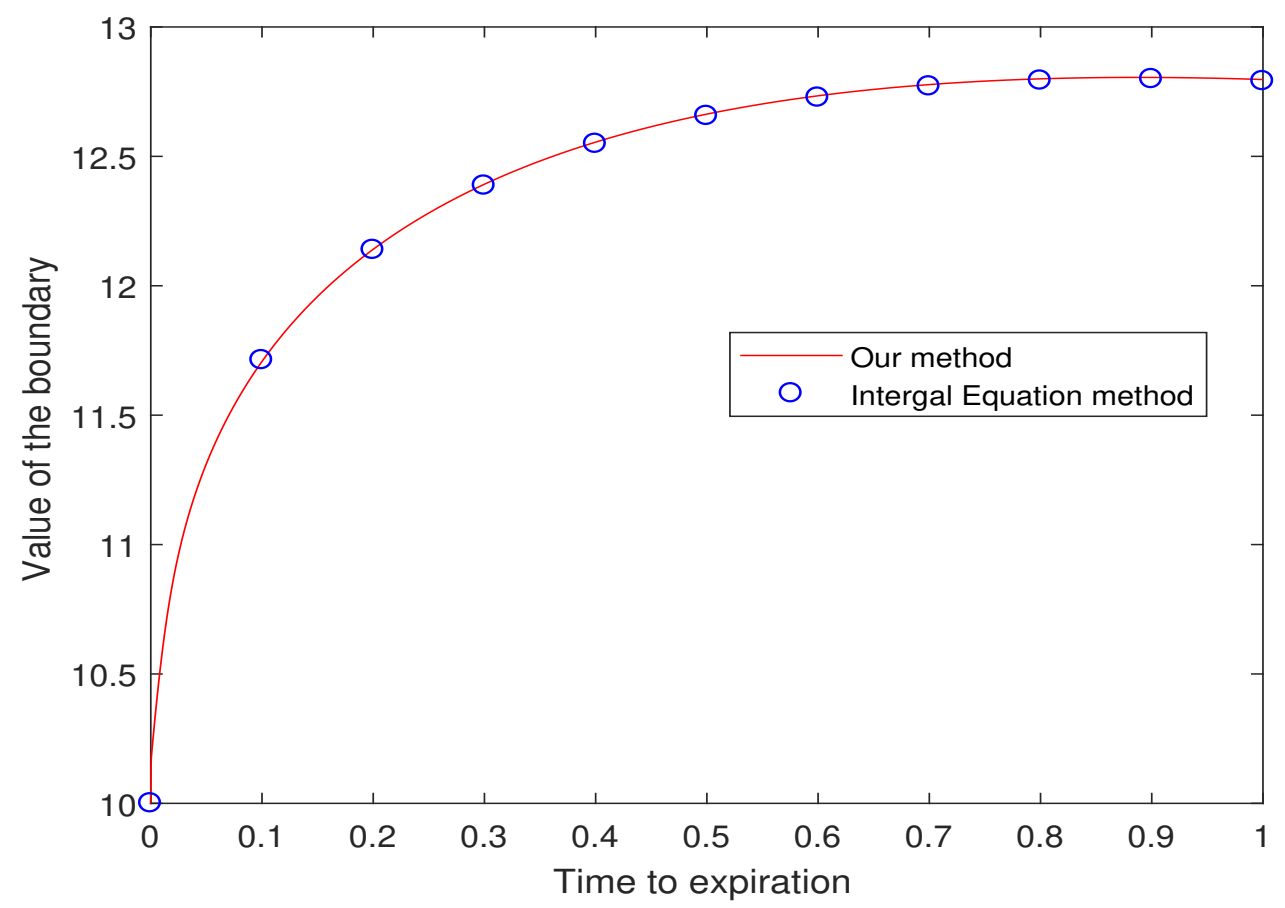

Figure 2: The comparison of the optimal conversion boundary obtained by our method and that from the integral equation method [45], at $v=0.1$.

shows the prices of the optimal conversion boundary at the current time, $t=0$, and one can easily observe that with the increase in the number of grid points, our results converge to the benchmark, as the relative error between the two prices are decreasing. If we turn to Figure 2, it is clear that both values match very well with each other, demonstrating the accuracy of our method for this case. Of course, we still need to check whether our approach works for the general case when the stochastic volatility is incorporated. Thus, the values of CBs obtained from our method are also compared with those generated through the Monte Carlo method, the results of which are presented in Figure 3. It can be easily noticed that both prices are point-wisely close to each other, which certainly reflects that our method is reliable. On the other hand, it should be point out that the average CPU time consumed by the Monte Carlo method, with 100 time steps and 500,000 sample paths, is 10.3828 seconds, while it only takes $7.9 \times 10^{-7}$ seconds to produce one price with the method introduced by this paper. Such a great difference certainly indicates that the 


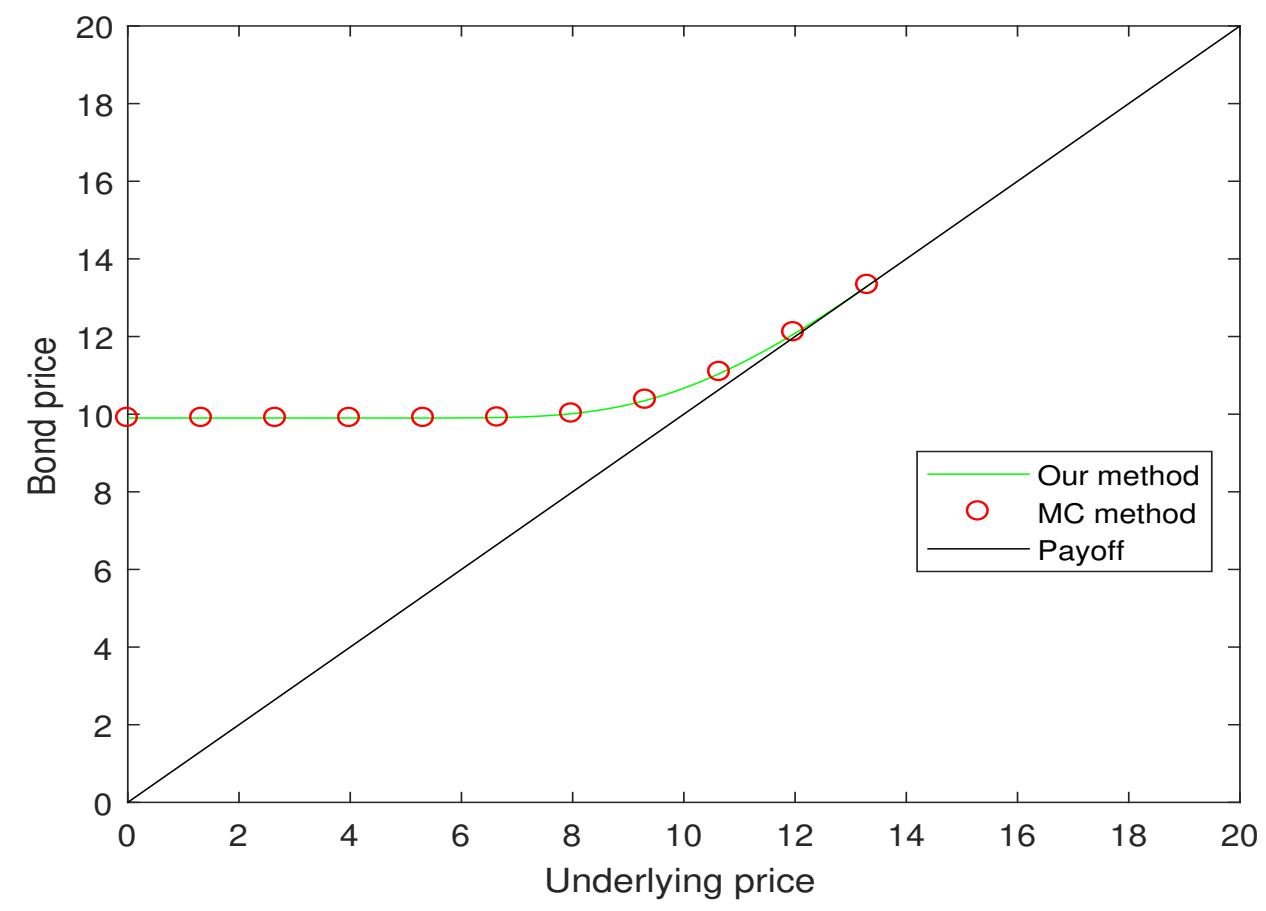

Figure 3: The comparison of the bond prices obtained by our method and those from the Monte Carlo method, at $t=0$ and $v=0.4$.

proposed predictor-corrector scheme is much more efficient.

With the confidence in our approach, we are now studying the properties of the optimal conversion boundary as well as the bond prices when the stochastic volatility is considered. Depicted in Figure 4 and Figure 5 are the values of the optimal conversion boundary with respect to the time to expiry and the volatility, respectively. An interesting phenomenon that can be observed in Figure 4 is that the optimal conversion boundary is not a monotonic increasing function of the time to expiry; it increases with the time to expiry when the time to expiry is small, and it will show a downward trend once the time to expiry is large enough. This is consistent with the theoretical result [41] that the value of the perpetual optimal conversion boundary equals to zero. From the financial point of view, it can be understood from the extreme case that when the time to expiry approaches infinity, the current value of the face value is almost zero, implying that it is meaningless to continue to hold the $\mathrm{CB}$ and the investor should convert it into stocks immediately. On the other 


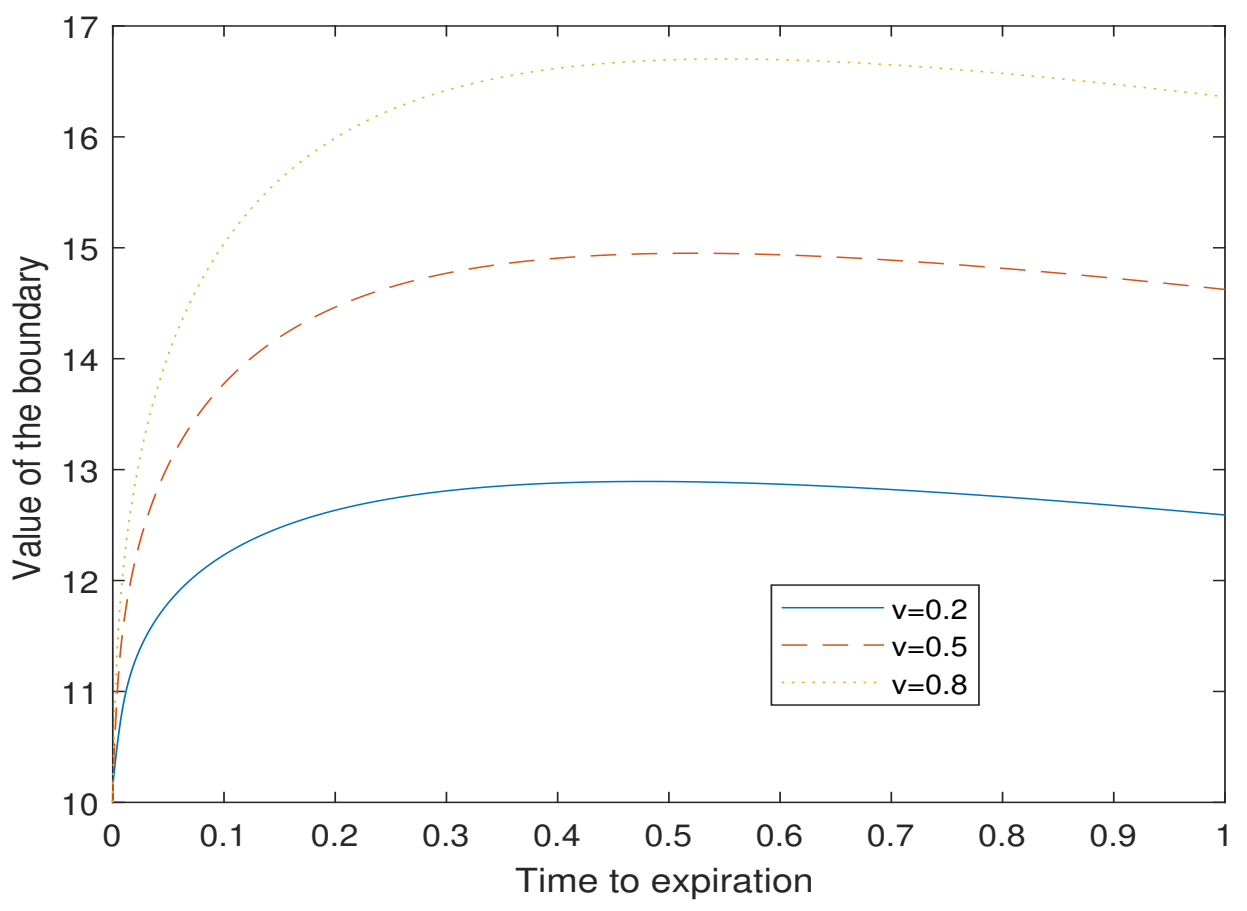

Figure 4: The optimal conversion price.

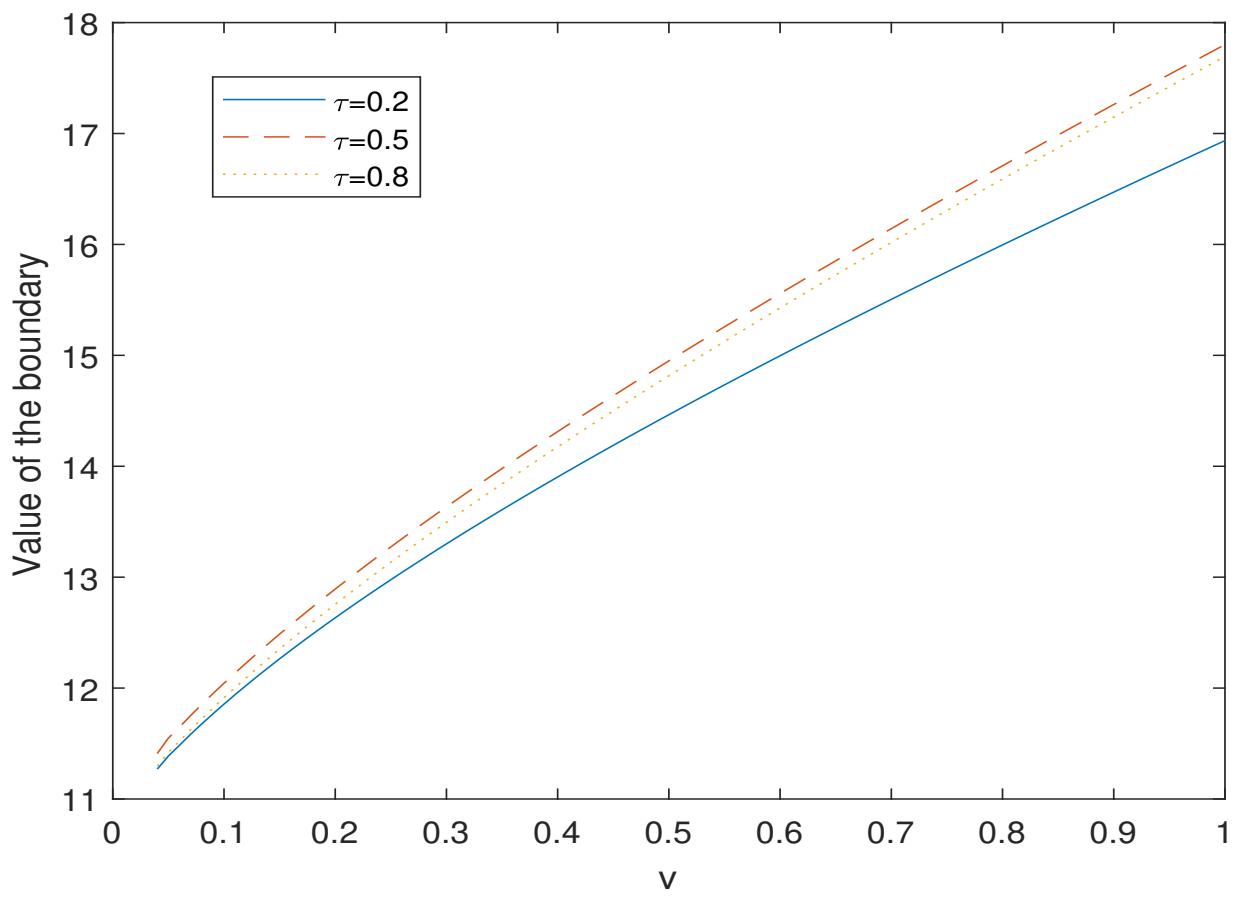

Figure 5: The optimal conversion price. 
hand, when we turn to Figure 5, it should be noted that the optimal conversion boundary is always a monotonic increasing function of the volatility, no matter the value of the time to expiry, which is reasonable since a higher volatility means a higher risk, leading to a higher premium of the CB.

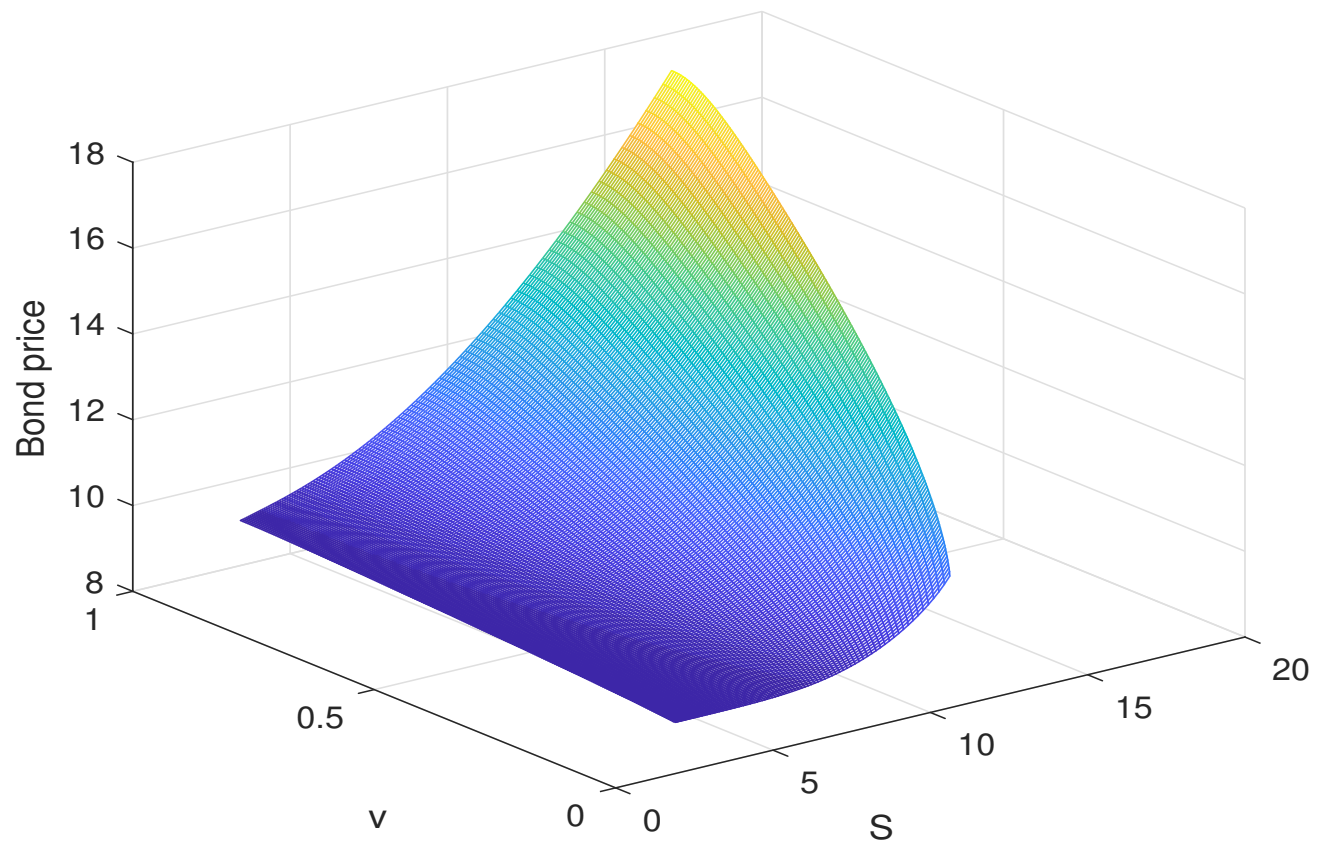

Figure 6: The bond price at $\tau=1$.

What is shown in Figure 6 is the change of the bond price with respect to the volatility and the underlying price, when the $\mathrm{CB}$ has not been converted into stocks. Clearly, no matter what the value of the underlying asset is, the bond price is a monotonic increasing function of the volatility as a larger volatility always implies a higher risk. Moreover, a higher value of the underlying asset leads to the larger slope of the bond price with respect to the volatility. In other words, the bond price is also an increasing function of the underlying asset, when the volatility is fixed, which is also clearly presented in Figure 7. This is financially meaningful since when the underlying asset price increases, there will be a higher probability for the holder to convert the bond, leading to the higher value of the bond. Another phenomenon that can be noticed here is that increasing the value of 


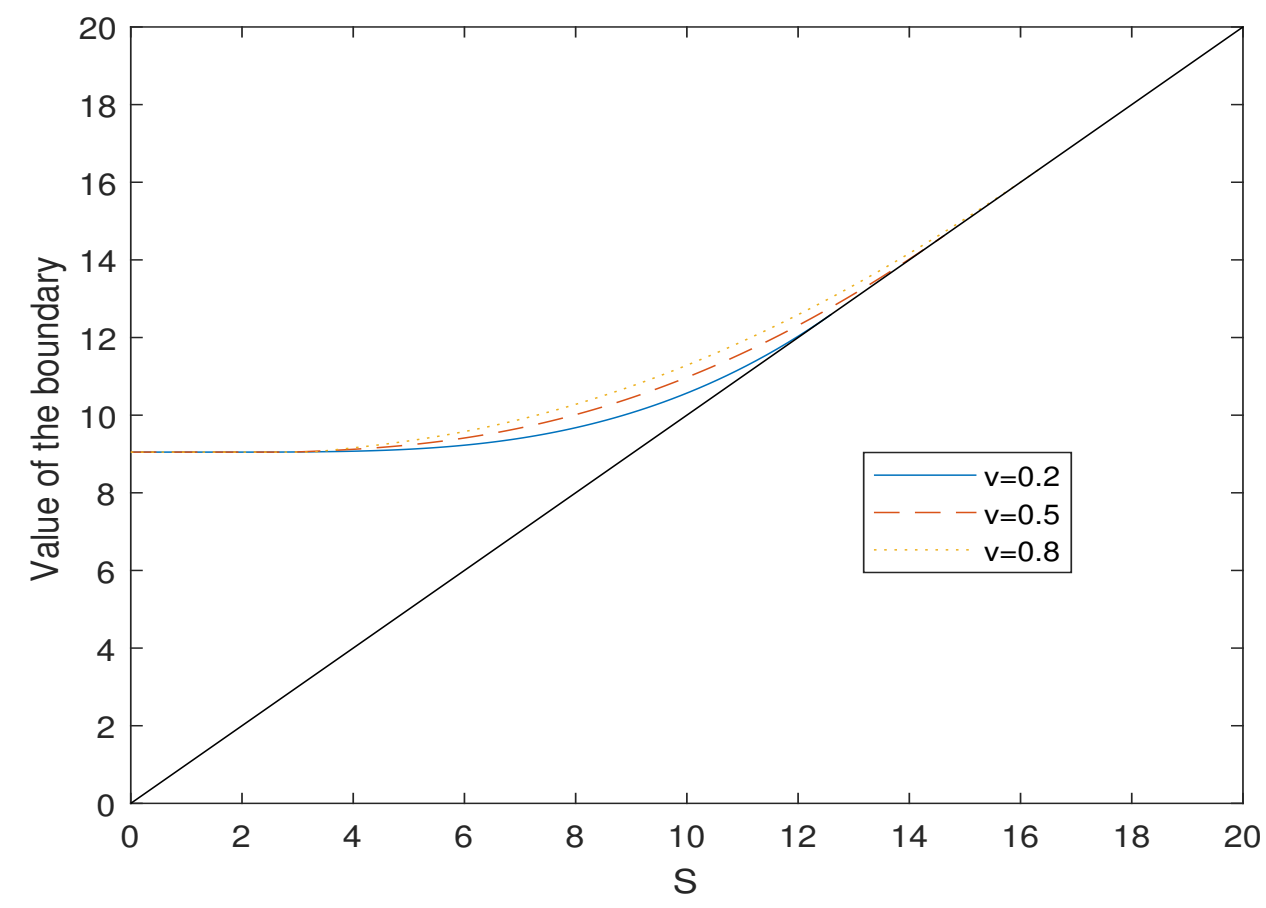

Figure 7: The bond price at $\tau=1$.

the volatility is actually increasing the value of the optimal conversion boundary, which confirms the result presented in Figure 5.

\section{Pricing convertible bonds with stochastic interest rate}

In this section, we study the pricing problem of the convertible bond with a stochastic interest rate model (CIR model). Given the fact that the PDE system in this section is very similar to that in the last section and the same predictor-corrector scheme will also be utilized here, the details on some tedious but very similar computational processes are thus omitted. 


\subsection{The PDE system and its numerical scheme}

We now begin by assuming that the stochastic interest rate satisfies the following SDE

$$
d r_{t}=\kappa\left(\eta-r_{t}\right) d t+\xi \sqrt{r_{t}} d W_{t}^{3}
$$

where $\kappa, \eta$ and $\xi$ are the mean reversion speed, the long term mean and the volatility of the interest rate, respectively, while $W_{3}$ is another standard Brownian motion. Due to the existence of $\sqrt{r_{t}}$, the value of the interest rate should also be non-negative, and thus the Feller condition should again be imposed here, which leads to $\kappa \eta>\frac{1}{2} \xi^{2}$. In addition, the dynamics of the underlying asset price is assumed as

$$
d S_{t}=\left(r_{t}-D_{0}\right) S_{t} d t+\sigma S_{t} d W_{t}^{1}
$$

which is the same as Equation (2.1) except that the constant volatility of the underlying asset is denoted as $\sigma$. The correlation between $W_{1}$ and $W_{3}$ is also represented by $\rho$, which can vary within $[-1,1]$. In this case, if the bond price is denoted as $U(S, r, t)$, its governing 
PDE system ${ }^{2}$ can be set up

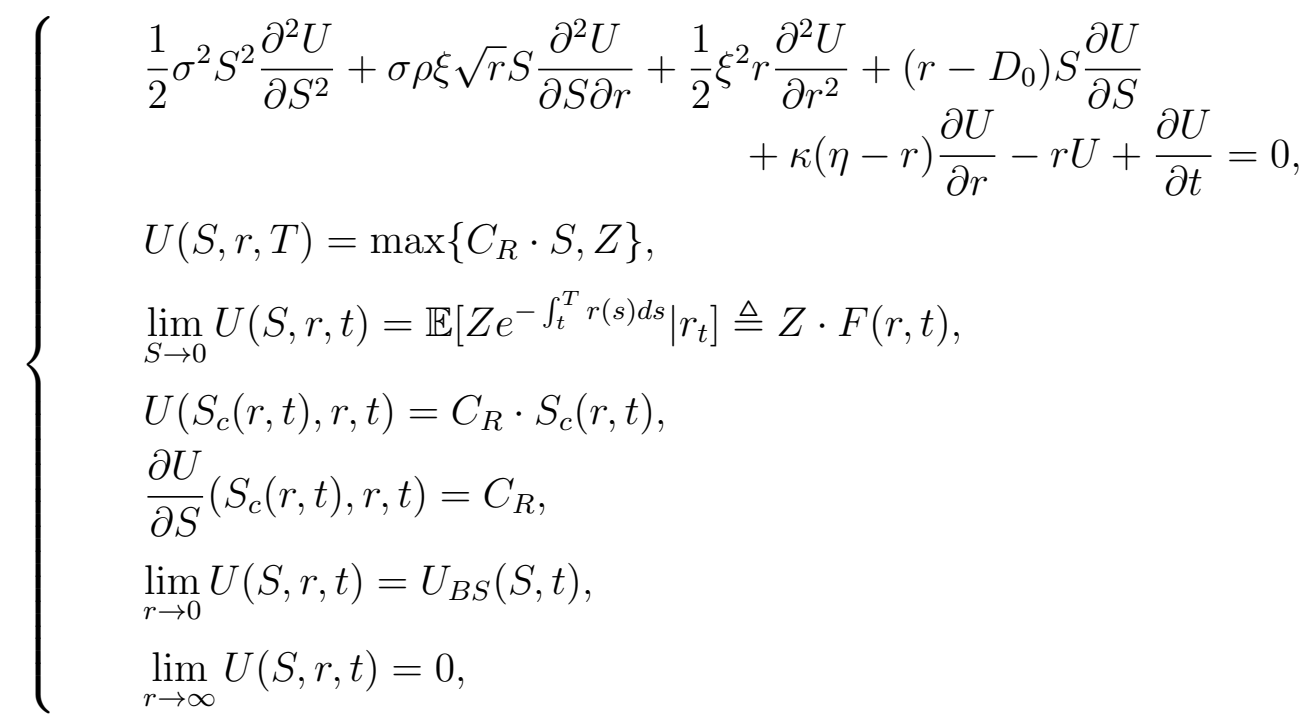

where $S_{c}(r, t)$ and $U_{B S}(S, t)$ are the optimal conversion boundary and the convertible bond price under the Black-Scholes model with $r=0$, respectively, and $F(r, t)$ satisfies the following PDE system

$$
\left\{\begin{array}{l}
\frac{\partial F}{\partial t}(r, t)+\kappa(\eta-r) \frac{\partial F}{\partial r}(r, t)+\frac{1}{2} \xi^{2} r \frac{\partial^{2} F}{\partial r^{2}}(r, t)-r F(r, t)=0 \\
F(r, T)=1
\end{array}\right.
$$

The solution to this PDE system can be found as

$$
F(r, t)=e^{A(t)-B(t) r}
$$

where

$$
\begin{aligned}
& A(t)=-\kappa \eta\left\{\frac{4}{(m-\kappa)(m+\kappa)} \ln \left[\frac{2 m+(m+\kappa)\left(e^{m(T-t)}-1\right)}{2 m}+\frac{2}{\kappa-m}(T-t)\right]\right\}, \\
& B(t)=\frac{2\left(e^{\sqrt{m(T-t)}}-1\right)}{2 m+(\kappa+m)\left(e^{m(T-t)}-1\right)},
\end{aligned}
$$

\footnotetext{
${ }^{2}$ The PDE can be directly derived using the Feynman-Kac theorem, and it is slightly different from the PDE discussed in [44] as the stochastic sources of the two problems are different. The boundary conditions in the two PDEs are also different as the domains of the underlying price are distinct, which is resulted from the same reason stated in the previous section.
} 
with $m=\sqrt{\kappa+2 \xi^{2}}$, the derivation of which can be found in [19]. Before we proceed further, it is necessary for us to explain the boundary conditions we gave in the system in the direction of $r$. On one hand, for the boundary condition at $r=0$, it needs to be pointed out that this boundary condition is not necessary if the Fichera function [17] along $r=0$ satisfies $\kappa \eta-\frac{\xi^{2}}{2} \geq 0$, while a boundary condition at $r=0$ is needed to close the system when $\kappa \eta<\frac{\xi^{2}}{2}$. Again, there is no need to impose the Feller condition, a boundary condition at $r=0$. However, the PDE system itself does not require the satisfaction of the Feller condition, and thus it is still necessary to present a suitable boundary condition at $r=0$. We adopt the bond price under the Black-Scholes model with $r=0$ as an approximation, which is based on an assumption that when $\mathcal{O}\left(\kappa \eta \frac{\partial U}{\partial r}\right)$ is much smaller than the order of the other terms in (3.3) when $r=0$, the resulting equation degenerates to the Black-Scholes equation. On the other hand, when the value of the risk-free interest rate goes to infinity, the best way to achieve the best return for an investor is leave the money in a risk-free bank account, which implies that no one would invest in a convertible bond, resulting in the bond value being equal to zero. It should also be noted that the boundary conditions in the direction of $S$ can be derived using a similar argument adopted for the case of stochastic volatility. The boundary value along $S=0$ is still equal to the expectation of discounted face value, but it takes a different form because the interest rate is now stochastic, making the discounting factor a random variable as well.

Now, applying the following transform

$$
V(S, r, t)=U(S, r, t)-\mathbb{E}\left[Z e^{-\int_{t}^{T} r(s) d s} \mid r_{t}\right]=U(S, r, t)-Z \cdot F(r, t),
$$


to the above PDE system yields

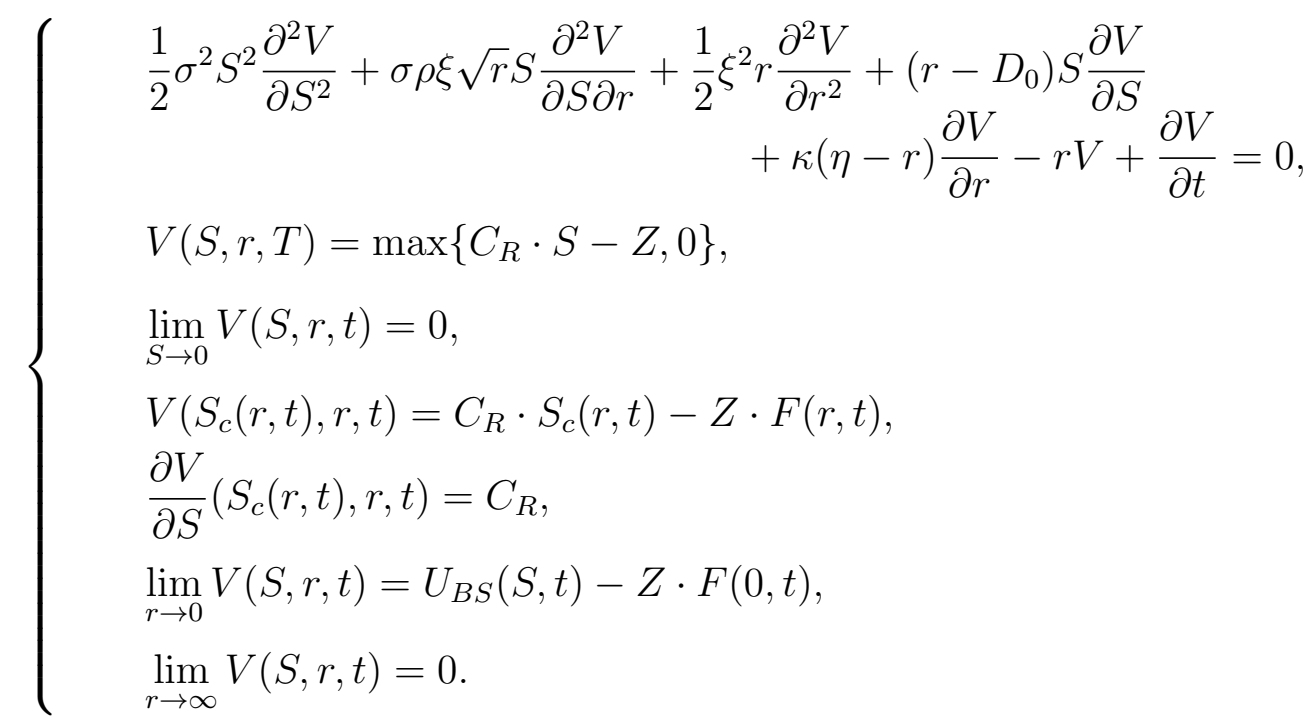

Then, in order to transform the target PDE system to a dimensionless one and also transform the free boundary problem into a fixed boundary one to facilitate the numerical computation, we make the following transformation

$$
\tau=T-t, \quad x=\ln \left(\frac{S}{S_{c}}\right)
$$

so that we have

$$
\left\{\begin{array}{l}
\mathcal{L}[V]=0, \\
V(x, r, 0)=\max \left\{C_{R} \cdot S_{c}(r, 0) \cdot e^{x}-Z, 0\right\}, \\
\lim _{x \rightarrow-\infty} V(x, r, \tau)=0, \\
V(0, r, \tau)=C_{R} \cdot S_{c}(r, \tau)-Z \cdot F(r, \tau), \\
\frac{\partial V}{\partial x}(0, r, \tau)=C_{R} \cdot S_{c}(r, \tau), \\
\lim _{r \rightarrow 0} V(x, r, \tau)=U_{B S}\left(S_{c}(0, \tau) \cdot e^{x}, \tau\right)-Z \cdot F(0, \tau), \\
\lim _{r \rightarrow \infty} V(x, r, \tau)=0,
\end{array}\right.
$$


where

$$
\mathcal{L}=a(r) \frac{\partial^{2}}{\partial x^{2}}+b(r) \frac{\partial^{2}}{\partial r^{2}}+c(r) \frac{\partial^{2}}{\partial x \partial r}+[d(r)+\lambda] \frac{\partial}{\partial x}+e(r) \frac{\partial}{\partial r}-r-\frac{\partial}{\partial \tau},
$$

with

$$
\begin{aligned}
a(r) & =\frac{1}{2} \sigma^{2}+\frac{1}{2} \xi^{2} r \zeta^{2}-\sigma \rho \xi \sqrt{r} \zeta, \\
b(r) & =\frac{1}{2} \xi^{2} r, \\
c(r) & =\sigma \rho \xi \sqrt{r}-\xi^{2} r \zeta, \\
d(r) & =-\frac{1}{2} \sigma^{2}+\frac{1}{2} \xi^{2} r \zeta^{2}-\frac{1}{2} \xi^{2} r \beta+r-D_{0}-\kappa(\eta-r) \zeta, \\
e(r) & =\kappa(\eta-r) .
\end{aligned}
$$

Here, we denote

$$
\zeta=\frac{1}{S_{c}} \cdot \frac{\partial S_{c}}{\partial r}, \quad \beta=\frac{1}{S_{c}} \cdot \frac{\partial^{2} S_{c}}{\partial r^{2}}, \quad \lambda=\frac{1}{S_{c}} \cdot \frac{\partial S_{c}}{\partial \tau} .
$$

Since this system is very similar to the last one, the FDE system is directly provided below without presenting all the details

$$
\left\{\begin{array}{l}
\frac{\partial V_{i, j}^{(n)}}{\partial \tau}=a_{j} \frac{\partial^{2} V_{i, j}^{(n)}}{\partial x^{2}}+b_{j} \frac{\partial^{2} V_{i, j}^{(n)}}{\partial r^{2}}+c_{j} \frac{\partial^{2} V_{i, j}^{(n)}}{\partial x \partial r}+\left[d_{j}+\lambda_{j}\right] \frac{\partial V_{i, j}^{(n)}}{\partial x}+e_{j} \frac{\partial V_{i, j}^{(n)}}{\partial r}-r_{j} V_{i, j}^{(n)}, \\
V_{i, j}^{(0)}=\max \left\{C_{R} \cdot S_{c}^{(0)}(j) e^{x_{i}}-Z, 0\right\} \\
V_{0, j}^{(n)}=0 \\
V_{N_{x}, j}^{(n)}=C_{R} \cdot S_{c}^{(n)}(j)-Z \cdot F^{(n)}(j), \\
\frac{3 V_{N_{x}, j}^{(n)}+V_{N_{x}-2, j}^{(n)}-4 V_{N_{x}-1, j}^{(n)}}{2 \Delta x}=C_{R} \cdot S_{c}^{(n)}(j), \\
V_{i, 0}^{(n)}=U_{B S}\left(S_{c}^{(n)}(0) \cdot e^{x_{i}}, \tau_{n}\right)-Z \cdot F^{(n)}(0), \\
V_{i, N_{r}}^{(n)}=0
\end{array}\right.
$$

with the divided finite domain being $\left\{\left(x_{i}, r_{j}, \tau_{n}\right) \mid x_{i}=x_{\min }+i \Delta x\right.$, for $i=0, \cdots, N_{x} ; r_{j}=$ $j \Delta r$, for $j=0, \cdots, N_{r} ; \tau_{n}=n \Delta \tau$, for $\left.n=0, \cdots, N_{\tau}\right\}$. Here, we have $\Delta x=-\frac{x_{\min }}{N_{x}}$, 
$\Delta r=\frac{r_{\max }}{N_{r}}$ and $\Delta \tau=\frac{T}{N_{\tau}}$, and the parameters are

$$
\begin{aligned}
a_{j} & =\frac{1}{2} \sigma^{2}+\frac{1}{2} \xi^{2} r_{j} \zeta_{j}^{2}-\sigma \rho \xi \sqrt{r_{j}} \zeta_{j}, \\
b_{j} & =\frac{1}{2} \xi^{2} r_{j}, \\
c_{j} & =\sigma \rho \xi \sqrt{r_{j}}-\xi^{2} r_{j} \zeta_{j}, \\
d_{j} & =-\frac{1}{2} \sigma^{2}+\frac{1}{2} \xi^{2} r_{j} \zeta_{j}^{2}-\frac{1}{2} \xi^{2} r_{j} \beta+r_{j}-D_{0}-\kappa\left(\eta-r_{j}\right) \zeta_{j}, \\
e_{j} & =\kappa\left(\eta-r_{j}\right),
\end{aligned}
$$

with

$$
\begin{aligned}
\zeta_{j} & =\frac{1}{S_{c}(j)} \cdot \frac{S_{c}(j+1)-S_{c}(j-1)}{2 \Delta r}, \\
\beta_{j} & =\frac{1}{S_{c}(j)} \cdot \frac{S_{c}(j+1)-2 S_{c}(j)+S_{c}(j-1)}{(\Delta r)^{2}}, \\
\lambda_{j} & =\frac{1}{S_{c}(j)} \cdot \frac{\partial S_{c}(j)}{\partial \tau} .
\end{aligned}
$$

In order to numerically solve the PDE system, we are now ready to set up the predictorcorrector scheme again with two steps. We will first briefly discuss how the predictor scheme can be established, which has the effect of linearizing the nonlinear PDE system (3.19). By using the boundary conditions at $x=0$

$$
\begin{aligned}
C_{R} \cdot S_{c}^{(n)}(j) & =\frac{3 V_{N_{x}, j}^{(n)}+V_{N_{x}-2, j}^{(n)}-4 V_{N_{x}-1, j}^{(n)}}{2 \Delta x}, \\
V_{N_{x}, j}^{(n)} & =C_{R} \cdot S_{c}^{(n)}(j)-Z \cdot F^{(n)}(j),
\end{aligned}
$$

we can further obtain

$$
\begin{aligned}
C_{R} \cdot S_{c}^{(n)}(j) & =\frac{3 V_{N_{x}, j}^{(n)}+V_{N_{x}-2, j}^{(n)}-4 V_{N_{x}-1, j}^{(n)}}{2 \Delta x} \\
\Rightarrow \quad C_{R} \cdot S_{c}^{(n)}(j) & =\frac{3\left(C_{R} \cdot S_{c}^{(n)}(j)-Z \cdot F^{(n)}(j)\right)+V_{N_{x}-2, j}^{(n)}-4 V_{N_{x}-1, j}^{(n)}}{2 \Delta x}
\end{aligned}
$$




$$
\begin{aligned}
& \Rightarrow \quad 2 \Delta x \cdot C_{R} \cdot S_{c}^{(n)}(j)=3\left(C_{R} \cdot S_{c}^{(n)}(j)-Z \cdot F^{(n)}(j)\right)+V_{N_{x}-2, j}^{(n)}-4 V_{N_{x}-1, j}^{(n)} \\
& \Rightarrow \quad(3-2 \Delta x) \cdot C_{R} \cdot S_{c}^{(n)}(j)=3 Z \cdot F^{(n)}(j)-V_{N_{x}-2, j}^{(n)}+4 V_{N_{x}-1, j}^{(n)} \\
& \Rightarrow \quad S_{c}^{(n)}(j)=\frac{3 Z \cdot F^{(n)}(j)-V_{N_{x}-2, j}^{(n)}+4 V_{N_{x}-1, j}^{(n)}}{(3-2 \Delta x) \cdot C_{R}} .
\end{aligned}
$$

Therefore, if we assume the value of the bond and that of the optimal conversion boundary at $n$th time step are known, then the optimal conversion price at $(n+1)$ th time step can be expressed

$$
S_{c}^{(n+1)}(j)=\frac{3 Z \cdot F^{(n+1)}(j)+4 V_{N_{x}-1, j}^{(n+1)}-V_{N_{x}-2, j}^{(n+1)}}{(3-2 \Delta x) \cdot C_{R}} .
$$

With the explicit Euler scheme and implicit Euler scheme being applied to the time derivative $\frac{\partial V_{i, j}^{(n)}}{\partial \tau}$ and $\frac{\partial S_{c}^{(n)}(j)}{\partial \tau}$, respectively, the following formulation can be obtained

$$
\begin{gathered}
V_{i, j}^{(n+1)}=V_{i, j}^{(n)}+\Delta \tau\left\{a_{j} \frac{\partial^{2} V_{i, j}^{(n)}}{\partial x^{2}}+b_{j} \frac{\partial^{2} V_{i, j}^{(n)}}{\partial r^{2}}+c_{j} \frac{\partial^{2} V_{i, j}^{(n)}}{\partial x \partial r}+\left[d_{j}+\frac{S_{c}^{(n+1)}(j)-S_{c}^{(n)}(j)}{\Delta \tau \cdot S_{c}^{(n)}(j)}\right] \frac{\partial V_{i, j}^{(n)}}{\partial x}\right. \\
\left.+e_{j} \frac{\partial V_{i, j}^{(n)}}{\partial r}\right\}-r_{j} \Delta \tau V_{i, j}^{(n)}, i=N_{x}-2, N_{x}-1 .
\end{gathered}
$$

As a result, the representation of the predicted optimal conversion price at $(n+1)$ th time step, $\tilde{S}_{c}^{(n+1)}(j)$, should be

$$
\tilde{S}_{c}^{(n+1)}(j)=\frac{3 Z \cdot F^{(n+1)}(j)+\mathcal{F}\left[4 V_{N_{x}-1, j}^{(n)}-V_{N_{x}-2, j}^{(n)}\right]}{(3-2 \Delta x) C_{R}-\frac{\delta_{x}\left(4 V_{N_{x}-1, j}^{(n)}-V_{N_{x}-2, j}^{(n)}\right)}{S_{c}^{(n)}(j)}}
$$

where

$$
\mathcal{F}=\mathbb{I}+\Delta \tau\left[a_{j} \delta_{x x}+b_{j} \delta_{r r}+c_{j} \delta_{x r}+\left(d_{j}-\frac{1}{\Delta \tau}\right) \delta_{x}+e_{j} \delta_{r}\right]-r_{j} \Delta \tau
$$

Here, all the derivatives are again replaced by the corresponding $\delta_{*}$. Hence, the predicted convertible bond price at $x=0, \tilde{V}_{N_{x}, j}^{(n+1)}$, is

$$
\tilde{V}_{N_{x}, j}^{(n+1)}=C_{R} \cdot \tilde{S}_{c}^{(n+1)}(j)-Z \cdot F^{(n+1)}(j)
$$


By now, the numerical scheme for the prediction step, where the optimal conversion price is predicted via Equation (3.34), and the bond price at $x=0$ is predicted through Equation (3.35), have been obtained, and the next step is to correct the values of $S_{c}^{(n+1)}$ and $V_{N_{x}}^{(n+1)}$ by using the obtained $V_{N_{x}-2}^{(n+1)}$ and $V_{N_{x}-1}^{(n+1)}$ at the prediction step using the ADI technique.

We are now again presenting the numerical scheme for the correction step directly, while omitting the details for the derivation. In order to apply the ADI method, the FDE also needs to be reformulated as

$$
\left(\mathbb{I}-\phi A_{1}\right)\left(\mathbb{I}-\phi A_{2}\right) V^{(n+1)}=\left[\mathbb{I}+A_{0}+(1-\phi) A_{1}+A_{2}\right] V^{(n)}-\left(\mathbb{I}-\phi A_{1}\right) \phi A_{2} V^{(n)},
$$

where the definitions of all operators, $A_{0}, A_{1}$ and $A_{2}$, are left in Appendix D. For the adopted D-R method, two steps should be taken into consideration. Firstly, we should fix the $r$ direction, and a intermediate value, $Y$, satisfies the following equation

$$
\left(\mathbb{I}-\phi A_{1}\right) Y=\left[\mathbb{I}+A_{0}+(1-\phi) A_{1}+A_{2}\right] V^{(n)},
$$

should be determined. Simplifying this equation leads to

$$
\mathbb{A} Y_{j}=P_{j}+\mathbb{B} x_{j}
$$

with the definitions of $\mathbb{A}, Y_{j}, P_{j}$ and $\mathbb{B} x_{j}$ left in Appendix E, and solving the set of equations here will give the value of $Y$. Once the value of $Y$ is known, the value of $V^{(n+1)}$ can be obtained from the following equation

$$
\left(\mathbb{I}-\phi A_{2}\right) V^{(n+1)}+\phi A_{2} V^{(n)}=Y
$$

with the $x$ direction being fixed. To deal with the above equation, it is again transformed 
into a set of equations

$$
\mathbb{C} V_{i}^{(n+1)}=Q_{i}+\mathbb{B} r_{i}
$$

with the details of $\mathbb{C}, V_{i}^{(n+1)}, Q_{i}$ and $\mathbb{B} r_{i}$ left in Appendix F. Of course, it is also easy to show that solving Equation (3.38) and Equation (3.40) means solving the initial Equation (3.36). In order to numerically implement our scheme, we need to make it clear how to calculate the boundary value of the intermediate value $Y$, which is presented as

$$
\begin{aligned}
Y_{0} & =0, \\
Y_{N_{x}} & =\left(\mathbb{I}-\phi A_{2}\right) \tilde{V}_{N_{x}}^{(n+1)}+\phi A_{2} V_{N_{x}}^{(n)} \\
& =\left(\mathbb{I}-\phi A_{2}\right)\left(C_{R} \cdot \tilde{S}_{c}^{(n+1)}-Z \cdot F^{(n+1)}\right)+\phi A_{2} V_{N_{x}}^{(n)},
\end{aligned}
$$

with the use of the predicted value of $\tilde{S}_{c}^{(n+1)}$. With the numerical scheme being established for the PDE system governing the bond price under the stochastic interest rate model, we are now ready to conduct numerical experiments, the details of which are presented in the following subsection. It should be pointed out that the implementation of this scheme is very similar to that of the one for the case of stochastic volatility, and thus the schematic flow chart for this case is omitted here as it can be similarly established as Figure 1.

\subsection{Convergence of the proposed scheme}

According to the Lax Equivalence Theorem [36], the the convergence of a numerical scheme is equivalent to its consistency and stability. Although the consistency of our approach is very straightforward to prove and thus details are omitted, the proof of stability is not so trivial, since we are applying a hybrid finite difference scheme to a problem with variable coefficients. Following the discussion in [44], the stability requirements of the explicit Euler scheme Equation (3.32) and the ADI scheme Equations (3.37) and (3.39) is presented in this subsection, based on which a conditional stability of the predictor-corrector scheme is 
established, using the local von Neumann stability analysis.

Although the von Neumann stability analysis is often restricted to problems with constant coefficients, the conditions for the stability of constant coefficient schemes can be used to impose stability conditions for the same scheme applied to equations with variable coefficients. This is mainly because instability is essentially a local phenomenon with the high frequency modes being the most unstable ones that would result in the collapse of a numerical scheme [36]. In this case, for the stability of a variable coefficient problem, one would only need to discuss frozen coefficient problems with constant coefficients obtained by fixing the coefficients at their values attained at each grid point in the computational domain. The target variable coefficient problem can be regarded as stable if each frozen coefficient problem is stable [36]. For simplicity, we do not take into account the overall effect of the boundary conditions between sub-domains in the following discussion.

As a prior step of the frozen coefficient technique, we need to "freeze" all the coefficients of the governing equation in (3.19) across the whole computational domain for a typical node $(i, j, n)$ so that they can be treated as constants. Thus, we just need to first discuss the stability condition of the D-R method when it is applied to a two-dimensional convectiondiffusion equation with constant coefficients, i.e.,

$$
\frac{\partial V}{\partial \tau}=a \frac{\partial^{2} V}{\partial x^{2}}+b \frac{\partial^{2} V}{\partial r^{2}}+c \frac{\partial^{2} V}{\partial x \partial r}+d \frac{\partial V}{\partial x}+e \frac{\partial V}{\partial r}-r V
$$

where $a, b$ and $r$ are all the positive. Following the standard procedure of von Neumann stability analysis, $V_{i, j}^{(n)}$ and $Y_{i, j}$ in Equations (3.37) and (3.39) should be expressed by $g^{n} e^{l i \varphi} e^{l j \theta}$ and $\tilde{g} g^{n} e^{l i \varphi} e^{l j \theta}$, respectively [36], where $g$ and $\tilde{g}$ are respectively the amplification factors of Equation (3.39) and Equation (3.37) with $\varphi, \theta \in[-\pi, \pi]$, and $l$ being the imaginary unit. As a result, Equations (3.37) and (3.39) are directly transformed into:

$$
\tilde{g}\left(1-\phi z_{1}\right)=1+z_{0}+(1-\phi) z_{1}+z_{2}
$$




$$
g\left(1-\phi z_{2}\right)=\tilde{g}-\phi z_{2}
$$

which further yields

$$
g=1+\frac{z_{0}+z_{1}+z_{2}}{\left(1-\phi z_{1}\right)\left(1-\phi z_{2}\right)}
$$

where

$$
\begin{aligned}
& z_{1}=-\frac{4 a \Delta \tau}{\Delta x^{2}} \sin ^{2} \frac{\varphi}{2}-\frac{r \Delta \tau}{2}+l \frac{d \Delta \tau}{\Delta x} \sin \varphi \\
& z_{2}=-\frac{4 b \Delta \tau}{\Delta r^{2}} \sin ^{2} \frac{\theta}{2}-\frac{r \Delta \tau}{2}+l \frac{e \Delta \tau}{\Delta r} \sin \theta \\
& z_{0}=-\frac{c \Delta \tau}{\Delta x \Delta r} \sin \varphi \sin \theta
\end{aligned}
$$

after some algebraic manipulations.

Lemma 3.1 (i) If the coefficients $a, b, c, d, e, r$ are chosen such that

$$
\left|z_{0}\right| \leq 2 \sqrt{\mathcal{R}\left(z_{1}\right) \mathcal{R}\left(z_{2}\right)}
$$

then for $\phi \geq \frac{1}{2}$, the D-R method (Equations (3.37) and (3.39)) is unconditionally stable, i.e., $|g| \leq 1$.

(ii)Assuming that the coefficients satisfy $c^{2} \leq 4 a b$, the fully explicit scheme (Equations (3.37) and (3.39) with $\phi=0)$, is stable if and only if

$$
\Delta \tau \leq \frac{1}{\frac{2 a}{\Delta x^{2}}+\frac{2 b}{\Delta r^{2}}}
$$

Proof. See [44].

Theorem 1 Assuming that the optimal exercise price is known in advance, and all the variable coefficients are both bounded and sufficiently smooth, the D-R method that is applied to the corrector (Equations (3.37) and (3.39)) is unconditionally stable ( $\left.\phi \geq \frac{1}{2}\right)$.

Proof. See [44]. 
Theorem 2 Assuming that the optimal exercise price is known in advance, and all the variable coefficients are both bounded and sufficiently smooth, the fully explicit scheme that is applied to the predictor Equation (3.32) $(\phi=0)$ is stable if and only if

$$
\Delta \tau \leq \frac{2}{a_{1}+a_{2}}
$$

where

$$
\begin{aligned}
a_{1} & =\frac{2 \sigma^{2}-4 \sigma \rho \xi \sqrt{r_{\max }} \zeta_{\min }+2 \xi^{2} r_{\max } \zeta_{\min }^{2}}{(\Delta x)^{2}} \\
a_{2} & =\frac{2 \xi^{2} r_{\max }}{(\Delta r)^{2}}
\end{aligned}
$$

(Here, we further assume that the correlation factor $\rho \geq 0$.)

Proof. By making use of the second part of Lemma 3.1 as well as the frozen coefficient technique, we only need to show that, the constraints $c^{2} \leq 4 a b$, and $\Delta \tau \leq \frac{1}{\frac{2 a}{\Delta x^{2}}+\frac{2 b}{\Delta r^{2}}}$ are both satisfied at each point across the whole computational domain, if

$$
\Delta \tau \leq \frac{2}{a_{1}+a_{2}}
$$

Recall that $a, b$ and $c$ in our case are defined as

$$
\begin{aligned}
a & =\frac{1}{2} \sigma^{2}+\frac{1}{2} \xi^{2} r \zeta^{2}-\sigma \rho \xi \sqrt{r} \zeta \\
b & =\frac{1}{2} \xi^{2} r \\
c & =\sigma \rho \xi \sqrt{r}-\xi^{2} r \zeta
\end{aligned}
$$

since

$$
\begin{aligned}
4 a b-c^{2} & =4 \cdot\left(\frac{1}{2} \sigma^{2}+\frac{1}{2} \xi^{2} r \zeta^{2}-\sigma \rho \xi \sqrt{r} \zeta\right) \cdot \frac{1}{2} \xi^{2} r-\left(\sigma \rho \xi \sqrt{r}-\xi^{2} r \zeta\right)^{2} \\
& =\left(\sigma^{2}+\xi^{2} r \zeta-2 \sigma \rho \xi \sqrt{r} \zeta\right) \xi^{2} r-\sigma^{2} \rho^{2} \xi^{2} r-\xi^{4} r^{2} \zeta^{2}+2 \sigma \rho \xi^{3} \zeta r^{3 / 2}
\end{aligned}
$$




$$
\begin{aligned}
& =\sigma^{2} \xi^{2} r-\sigma^{2} \rho^{2} \xi^{2} r \\
& =\sigma^{2} \xi^{2} r\left(1-\rho^{2}\right) \\
& \geq 0
\end{aligned}
$$

all the frozen coefficient problems are stable if and only if

$$
\Delta \tau \leq \frac{1}{\frac{2 a_{\max }}{\Delta x^{2}}+\frac{2 b_{\max }}{\Delta r^{2}}}
$$

Given that the optimal exercise price $S_{c}(r, \tau)$ is a monotonic decreasing function of $r$, and all the coefficients are assumed to be bounded, there must exist a $\zeta_{\text {min }}$, such that $\zeta_{\min } \leq \zeta_{j} \leq 0$, implying that $\frac{4 a_{\max }}{(\Delta x)^{2}}=a_{1}, \frac{4 b_{\max }}{(\Delta r)^{2}}=a_{2}$. This has completed the proof.

It should be remarked that the stability condition of the fully explicit scheme depends heavily on the value of $\xi$, and more steps in the time direction are needed when $\xi$ is large.

Based on the discussion above, we are now able to present the stability condition of the predictor-corrector method, which is illustrated in the following theorem.

Theorem 3 If $g_{1}$ is the amplification factor of the explicit Euler scheme used in the predictor, and $g_{2}$ is the one of the ADI scheme used in the corrector, then the predictorcorrector method is stable if and only if $\left|g_{1} g_{2}\right| \leq 1+M \Delta \tau$.

Proof. The values that are obtained after the predictor will be expressed with tildes, and the subscripts in the $r$ direction or $x$ direction will be omitted for simplicity, in the following proof.

With

$$
\tilde{S}_{c}^{(n+1)}=\frac{3 Z \cdot F^{(n+1)}+4 \tilde{V}_{N_{x}-1}^{(n+1)}-\tilde{V}_{N_{x}-2}^{(n+1)}}{(3-2 \Delta x) \cdot C_{R}}
$$

it is not difficult to derive

$$
\left|\tilde{S}_{c}^{(n+1)}-\tilde{S}_{c}^{(0)}\right| \leq \frac{3 Z \cdot\left|F^{(n+1)}-F^{(0)}\right|+4\left|\tilde{V}_{N_{x}-1}^{(n+1)}-\tilde{V}_{N_{x}-1}^{(0)}\right|+\left|\tilde{V}_{N_{x}-2}^{(n+1)}-\tilde{V}_{N_{x}-2}^{(0)}\right|}{(3-2 \Delta x) \cdot C_{R}}
$$


Since $F^{(n+1)}$ is a constant, the predictor is stable if and only if the process of computing $\tilde{V}^{(n+1)}$ is stable.

By making use of Equation (3.32), we can formulate

$$
\tilde{V}^{(n+1)}=\left(\mathbb{I}+A_{0}^{*}+A_{1}^{*}+A_{2}^{*}\right) V^{(n)},
$$

where a star denotes the parameter $\phi=0$, and $V^{(n)}$ is obtained through solving

$$
\begin{aligned}
& \left(\mathbb{I}-\phi A_{1}\right) Y=\left[\mathbb{I}+A_{0}+(1-\phi) A_{1}+A_{2}\right] \tilde{V}^{(n-1)}, \\
& \left(\mathbb{I}-\phi A_{2}\right) V^{(n)}=Y-\phi A_{2} \tilde{V}^{(n-1)} .
\end{aligned}
$$

Here $\tilde{V}^{(n-1)}$ is obtained after the predictor of the $n$th time step. To apply the von Neumann analysis, all the variable coefficients are frozen as constant. Applying the Fourier transform on Equations (3.67) and (3.68)-(3.69) leads to

$$
\begin{aligned}
\hat{\tilde{V}}_{i, j}^{(n+1)} & =\left(1+z_{0}^{*}+z_{1}^{*}+z_{2}^{*}\right) \hat{V}_{i, j}^{(n)}=g_{1} \hat{V}_{i, j}^{(n)} \\
\hat{V}_{i, j}^{(n)} & =1+\frac{z_{0}+z_{1}+z_{2}}{\left(1-\phi z_{1}\right)\left(1-\phi z_{2}\right)} \hat{\tilde{V}}_{i, j}^{(n-1)}=g_{2} \hat{\tilde{V}}_{i, j}^{(n-1)}
\end{aligned}
$$

where $\hat{\tilde{V}}_{i, j}^{(n+1)}$ and $\hat{V}_{i, j}^{(n)}$ are the Fourier transform of $\tilde{V}_{i, j}^{(n+1)}$ and $V_{i, j}^{(n)}$, respectively. This directly yields

$$
\hat{\tilde{V}}_{i, j}^{(n+1)}=g_{1} g_{2} \hat{\tilde{V}}_{i, j}^{(n-1)}
$$

implying that the overall amplification factor $g$ for the predictor-corrector approach is actually $g=g_{1} g_{2}$.

Therefore, we can easily deduce that our method is stable if and only if

$$
\left|g_{1}\left(x_{i}, r_{j}, \tau_{n} ; \Delta \tau\right) g_{2}\left(x_{i}, r_{j}, \tau_{n} ; \Delta \tau\right)\right| \leq 1+M \Delta \tau
$$

where $M$ is a constant being independent of $\varphi, \theta$, as well as all the step sizes, and $i, j, n$ 
are defined over the whole computational domain. This has completed the proof.

\subsection{Numerical examples}

In this subsection, the numerical examples are presented with the same values of the corresponding parameters used in the last section. The only exception is that the constant volatility, $\sigma$, is assumed to be 0.4 in this section. Although the scheme for the case of stochastic interest rate is almost as same as that for the case of stochastic volatility, which has been confirmed as accurate in the last section, we still provide its verification here to make sure that the numerical results obtained in this section are correct.

Table 2: Convergence test on the optimal conversion boundary

\begin{tabular}{c|c|c|c|c}
\hline Interest rate value & $(N x, N r, N t)$ & ADI & IE & Relative error \\
\hline \multirow{3}{*}{$r=0.1$} & $(25,50,1250)$ & 14.6237 & & $5.82 \times 10^{-3}$ \\
& $(50,100,2500)$ & 14.5606 & 14.5390 & $1.49 \times 10^{-3}$ \\
& $(100,200,5000)$ & 14.5447 & & $3.88 \times 10^{-4}$ \\
\hline$r=0.2$ & $(25,50,1250)$ & 13.2321 & & $5.78 \times 10^{-3}$ \\
& $(50,100,2500)$ & 13.1750 & 13.1560 & $1.44 \times 10^{-3}$ \\
& $(100,200,5000)$ & 13.1606 & & $3.45 \times 10^{-4}$ \\
\hline$r=0.4$ & $(25,50,1250)$ & 10.8335 & & $5.75 \times 10^{-3}$ \\
& $(50,100,2500)$ & 10.7868 & 10.7715 & $1.42 \times 10^{-3}$ \\
& $(100,200,5000)$ & 10.7750 & & $3.17 \times 10^{-4}$ \\
\hline
\end{tabular}

First of all, a degenerate case with the interest rate being fixed is adopted as a special benchmark, and the values of the optimal conversion boundary obtained with our method are compared with those from the integral equation approach [45]. In specific, Table 2 displays the convergence of our optimal conversion boundary to the benchmark at the current time with the increase in the number of grid points, and the magnitude of the relative error between the two prices clearly demonstrates the accuracy of our approach. This is also confirmed by Figure 8, where it is shown that the values from both approaches agree very well with each other. Of course, the accuracy of the degenerate case is not adequate, and we still need to provide evidence on whether our approach also works for the general case with the presence of the stochastic interest rate. Therefore, Figure 9 


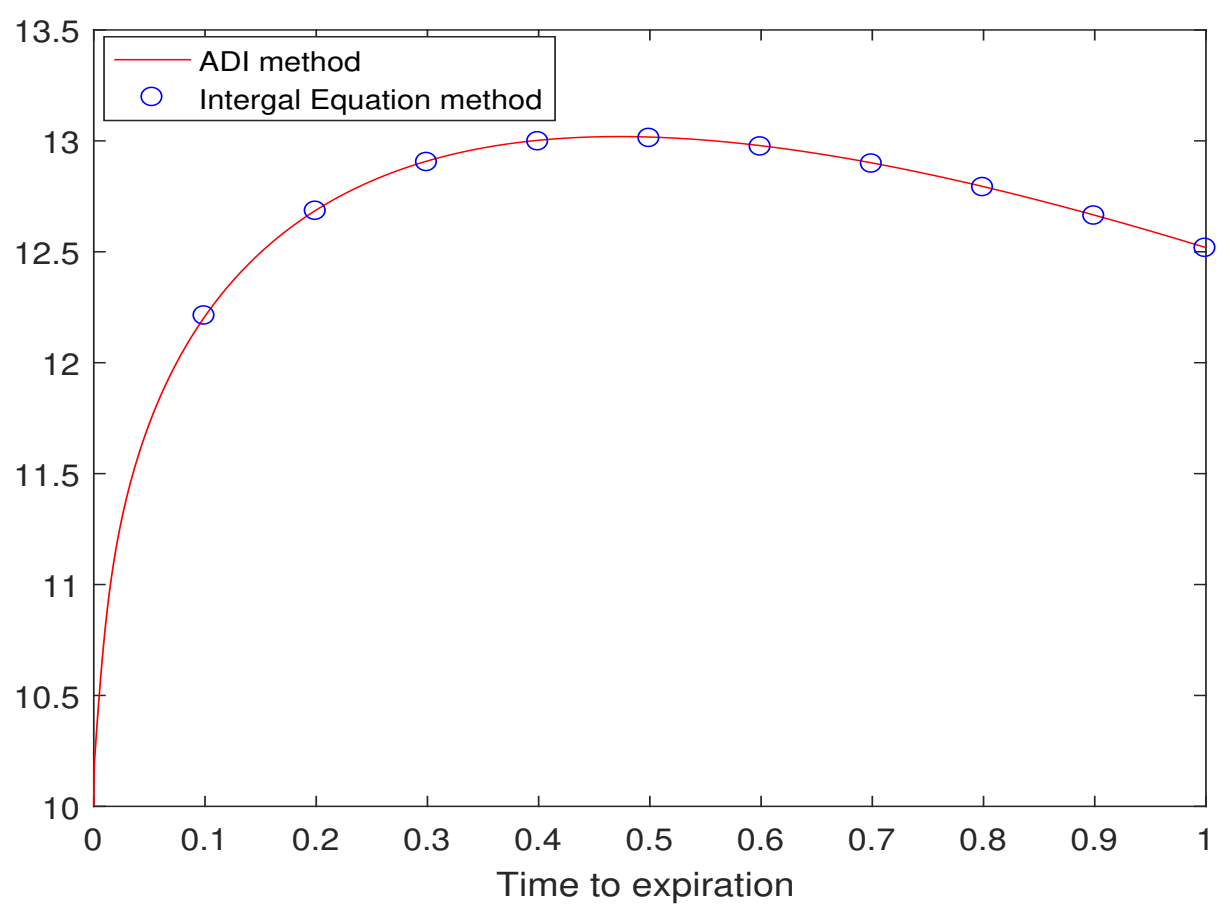

Figure 8: The comparison of the optimal conversion boundary obtained by our method and that from the integral equation method [45].

compares the bond prices from our method and those generated through the Monte Carlo simulation. The point-wise closeness between both prices clearly reveals the validity of our method. One may also be interested in the efficiency of our method, as the computational speed is one of the most important factors that affect practical applications. Therefore, it is worthwhile to mention here that it only costs $8.0 \times 10^{-7}$ seconds on average to produce one price with our method, while it takes the Monte Carlo method, with 100 time steps and 500,000 sample paths, 12.2600 seconds to produce the same price. This clearly shows the efficiency of our proposed predictor-corrector scheme.

Firstly, a similar phenomenon as shown in the case of stochastic volatility can be observed in Figure 10 that the optimal convertible price is not an increasing function of the time to expiry; the value of the optimal conversion boundary increases with the time to expiry initially, before it decreases. The main explanation for this is also the fact that the optimal conversion boundary of a perpetual $\mathrm{CB}$ is zero, as discussed in the case of 


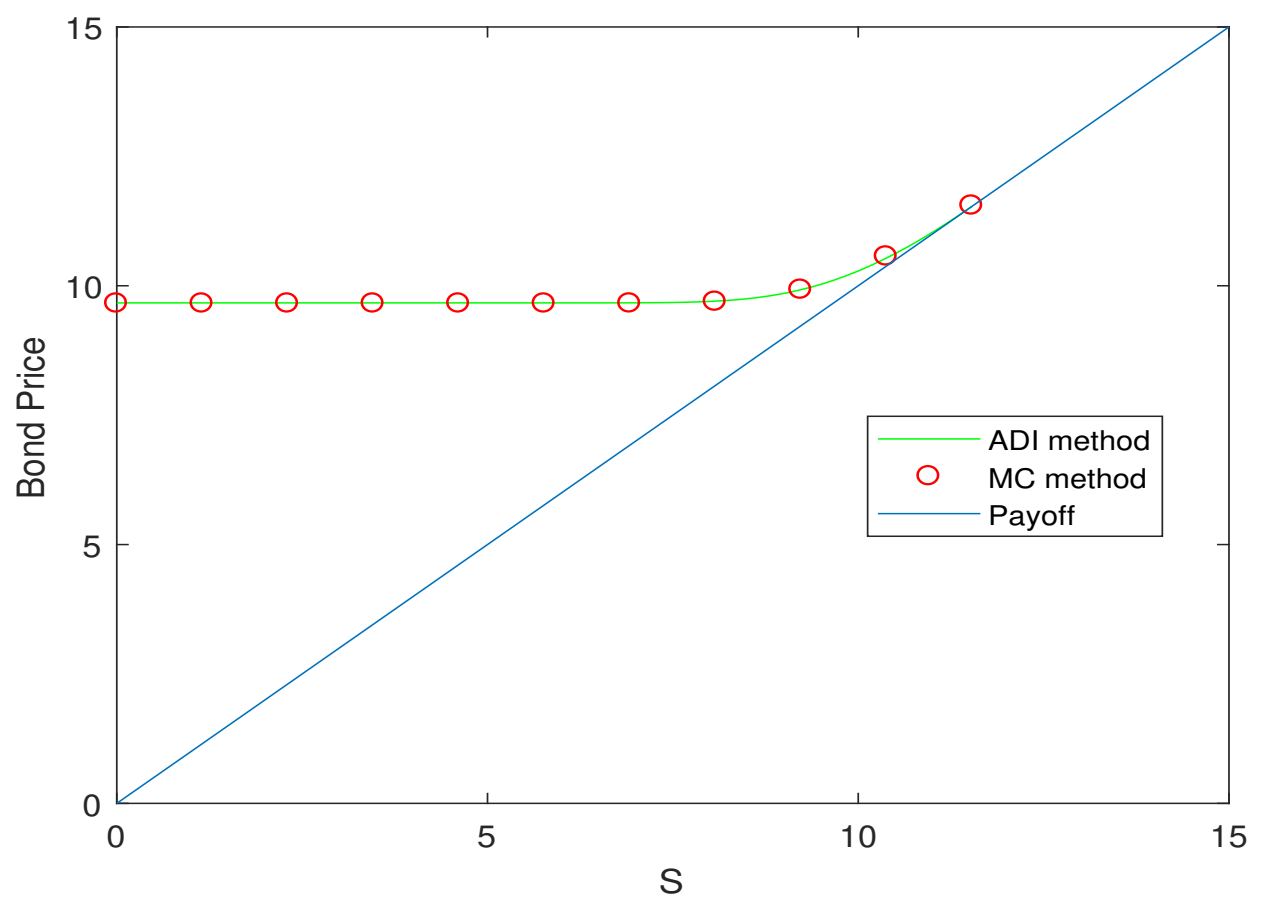

Figure 9: The comparison of the bond prices obtained by our method and those from the Monte Carlo method.

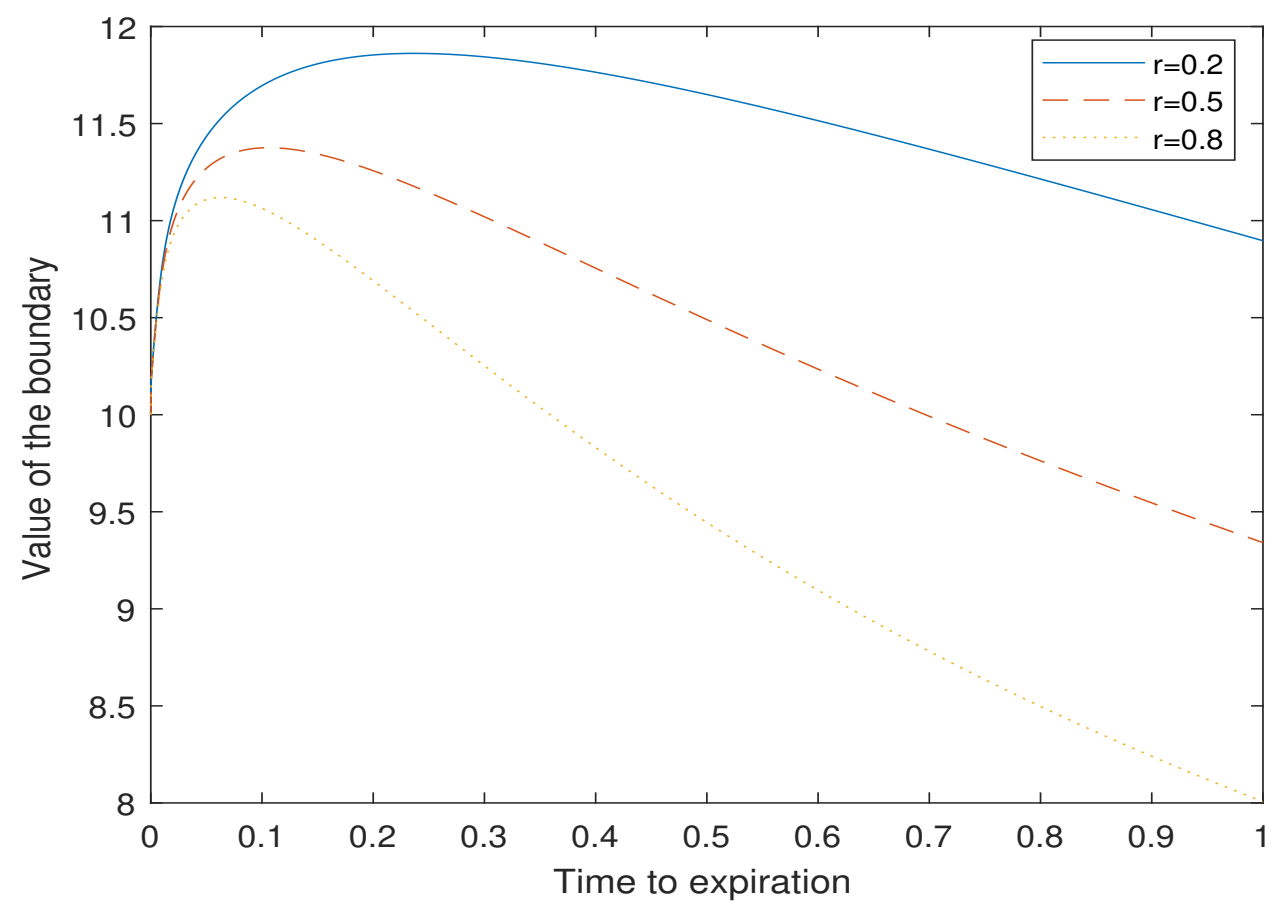

Figure 10: The optimal conversion price. 


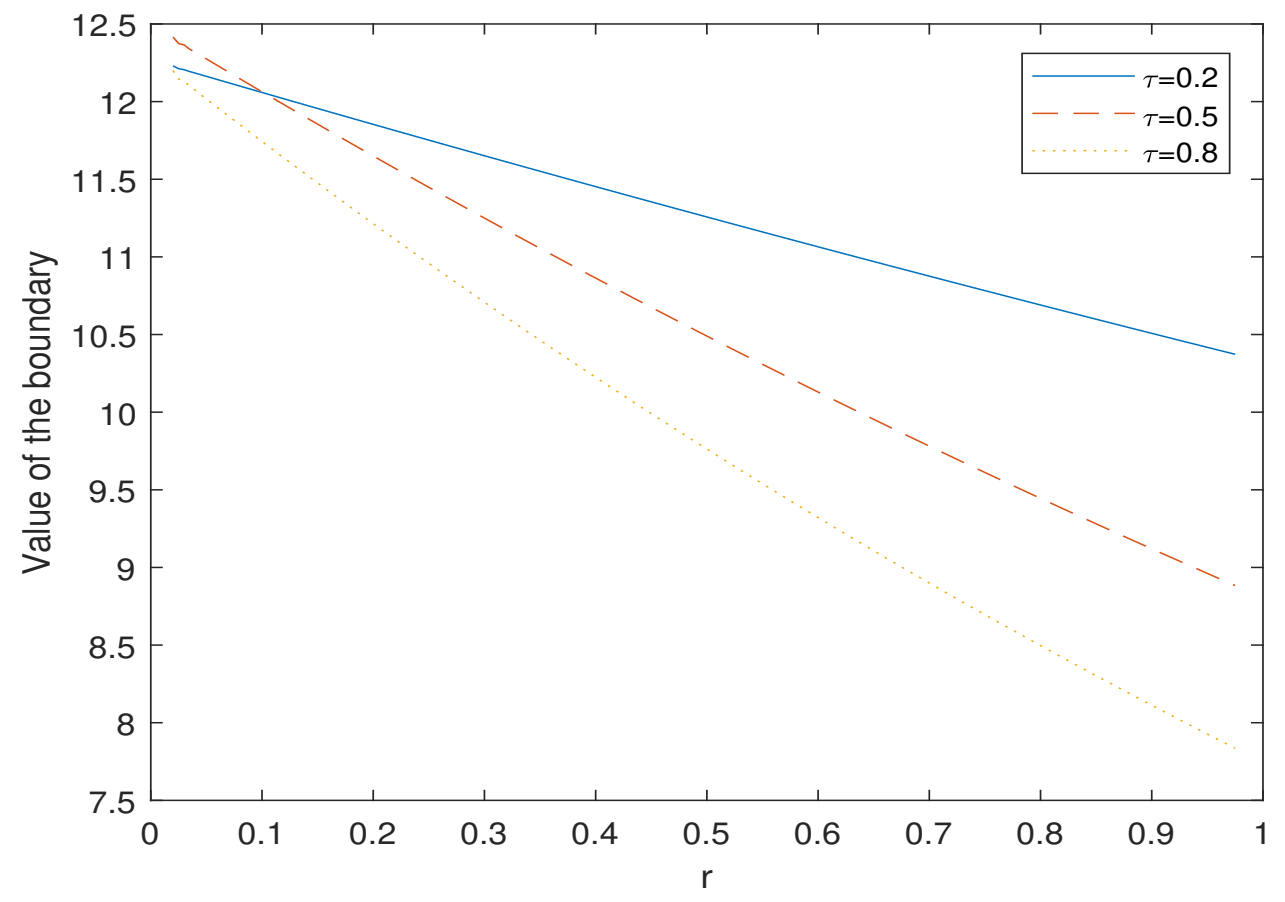

Figure 11: The optimal conversion price.

stochastic volatility. When we look at Figure 11, we can find that the value of the optimal conversion boundary is actually a decreasing function of the interest rate, no matter what the lifetime of the bond is. This is also financially reasonable since the higher the interest rate, the lower the present value of the face value will be, implying that the holder will choose to convert the bond at a smaller underlying price.

In Figure 12, the effects of the interest rate and the underlying price on the bond price with a certain time to expiry are demonstrated, and we again only consider the case when the $\mathrm{CB}$ has not been converted. When the interest rate is taken into consideration, it is not difficult to find that the price of the $\mathrm{CB}$ is a monotonic decreasing function with respect to the interest rate, which is reasonable since a higher value of the interest rate means that it is more incentive for the holder to leave their money in a risk-free environment than buying a risky bond, leading to a lower bond value. When we turn to the underlying price, the effect of which is displayed in Figure 13, it can be observed that the price of the convertible bond is actually an increasing function of the underlying price, which can be understood 


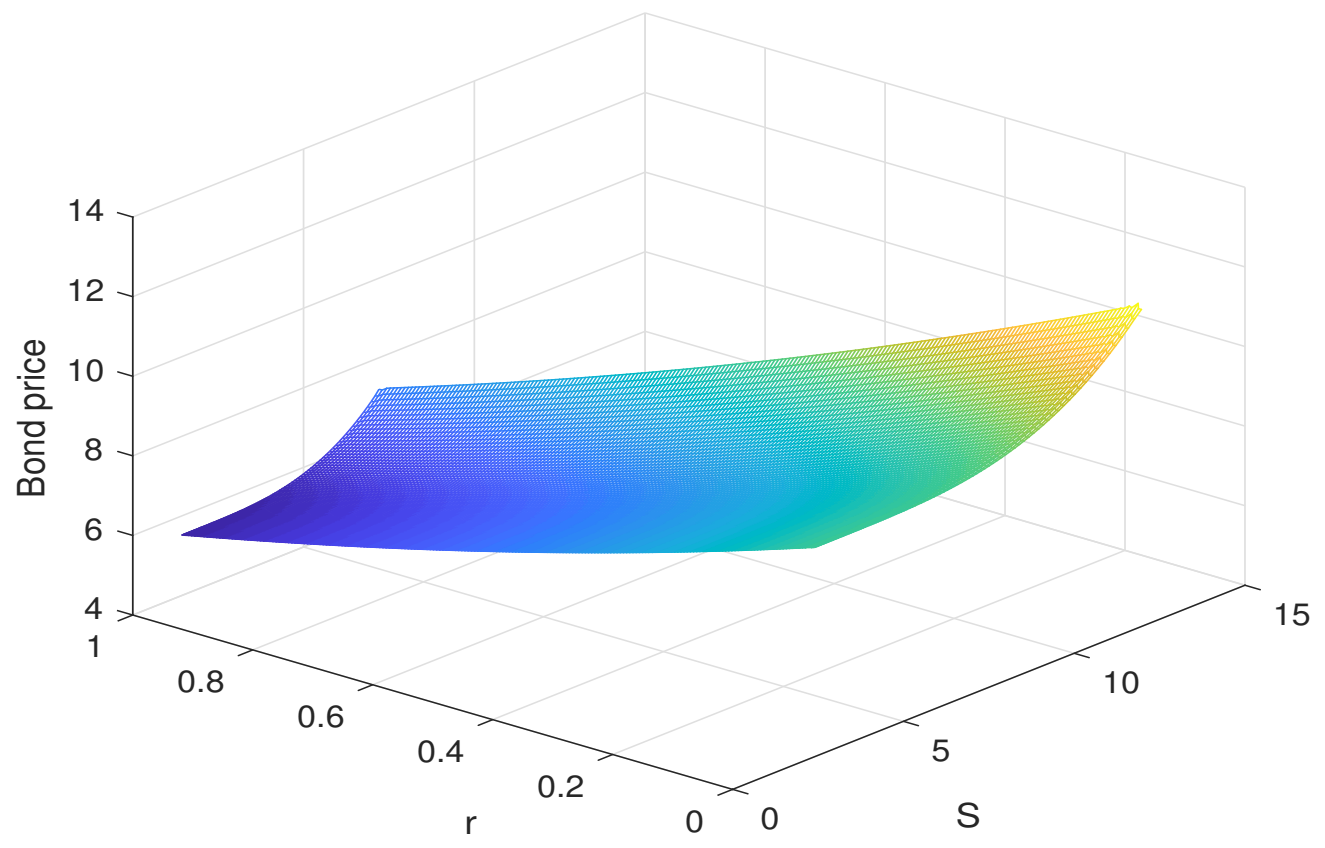

Figure 12: The bond price at $\tau=1$.

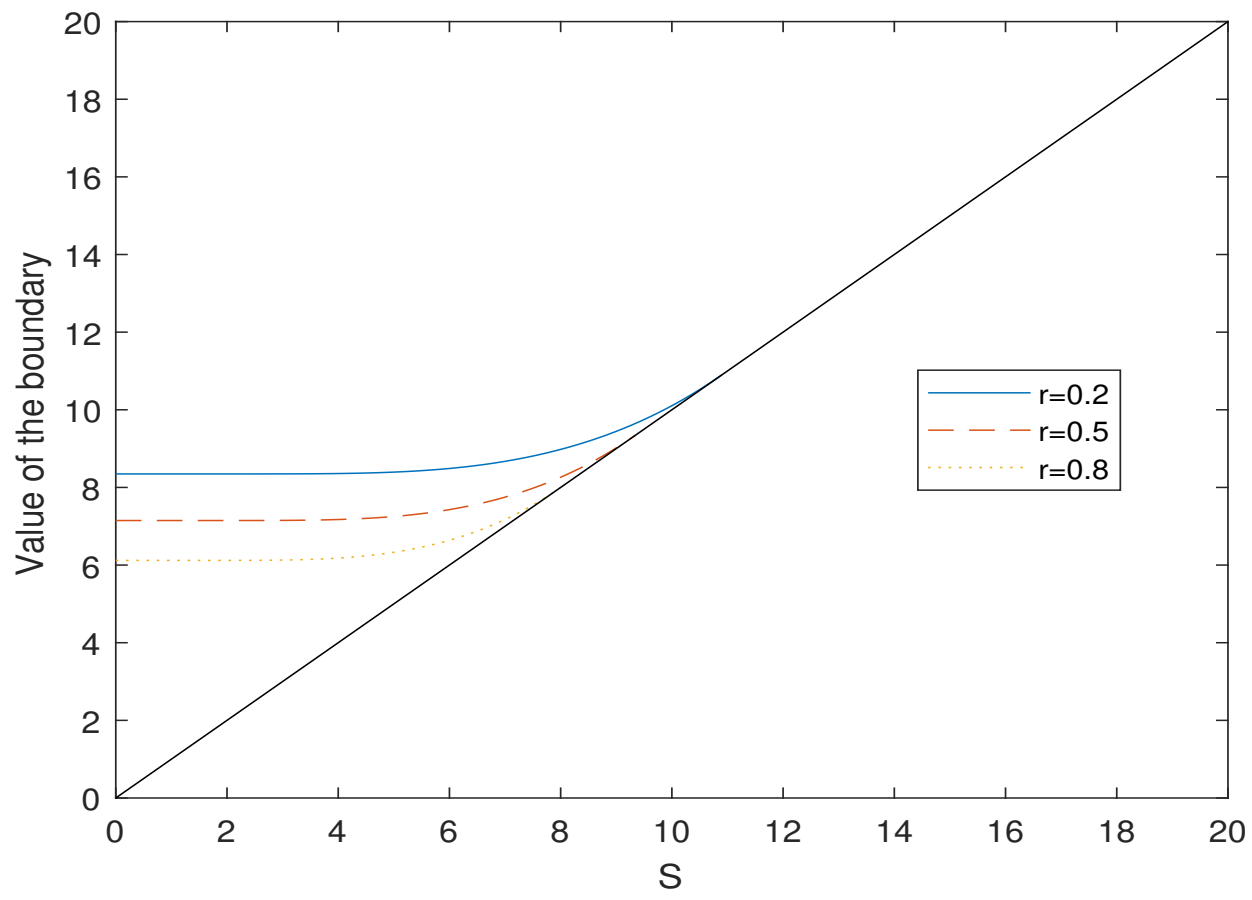

Figure 13: The bond price at $\tau=1$. 
with a similar reason as provided for the case of stochastic volatility.

\section{Conclusion}

In this paper, the pricing problems of convertible bonds with a stochastic volatility model and a stochastic interest rate model are considered, respectively. An efficient numerical scheme, the predictor-corrector scheme, is established for these two cases. Being able to provide the entire optimal conversion boundary as part of the solution procedure, this new approach requires no embedded iterations at all. Finally, numerical experiments are also carried out to show the reliability of our approach, and different properties of the convertible bond price as well as the optimal convertible boundary are investigated.

\section{Acknowledgments}

The first author would like to gratefully acknowledge the International Postgraduate Tuition Award as well as other research support provided by the University of Wollongong, where all the work presented in this paper was carried out during her Ph.D.

\section{Appendix}

\section{Appendix A}

Recall the FDE in our system

$$
\frac{\partial V_{i, j}^{(n)}}{\partial \tau}=a_{j} \delta_{x x} V_{i, j}^{(n)}+b_{j} \delta_{v v} V_{i, j}^{(n)}+c_{j} \delta_{x v} V_{i, j}^{(n)}+\left[d_{j}+\lambda_{j}\right] \delta_{x} V_{i, j}^{(n)}+e_{j} \delta_{v} V_{i, j}^{(n)}-r V_{i, j}^{(n)}
$$


with the implicit Euler scheme applied to the time derivative function, we can obtain

$$
\frac{V_{i, j}^{(n+1)}-V_{i, j}^{(n)}}{\Delta \tau}=a_{j} \delta_{x x} V_{i, j}^{(n+1)}+b_{j} \delta_{v v} V_{i, j}^{(n+1)}+c_{j} \delta_{x v} V_{i, j}^{(n+1)}+\left[d_{j}+\lambda_{j}\right] \delta_{x} V_{i, j}^{(n+1)}+e_{j} \delta_{v} V_{i, j}^{(n+1)}-r V_{i, j}^{(n+1)}
$$

If we define

$$
\begin{aligned}
A_{0} & =\Delta \tau \cdot c_{j} \delta_{x v} \\
A_{1} & =\Delta \tau\left[a_{j} \delta_{x x}+\left(d_{j}+\lambda_{j}\right) \delta_{x}-\frac{r}{2} \mathbb{I}\right] \\
A_{2} & =\Delta \tau\left[b_{j} \delta_{v v}+e_{j} \delta_{v}-\frac{r}{2} \mathbb{I}\right]
\end{aligned}
$$

then the PDE can be derived

$$
\left[\mathbb{I}-\left(A_{0}+A_{1}+A_{2}\right)\right] V_{i, j}^{(n+1)}=V_{i, j}^{(n)}+\mathcal{O}\left((\Delta \tau)^{2}\right) .
$$

Similarly, if the explicit Euler scheme used instead of the implicit one, we obtain

$$
\left[\mathbb{I}+\left(A_{0}+A_{1}+A_{2}\right)\right] V_{i, j}^{(n)}=V_{i, j}^{(n+1)}+\mathcal{O}\left((\Delta \tau)^{2}\right) .
$$

Therefore, the weighted average of these two scheme can be displayed

$$
\left[\mathbb{I}-\phi\left(A_{0}+A_{1}+A_{2}\right)\right] V_{i, j}^{(n+1)}=\left[\mathbb{I}+(1-\phi)\left(A_{0}+A_{1}+A_{2}\right)\right] V_{i, j}^{(n)}+\mathcal{O}\left((\Delta \tau)^{2}\right) .
$$

It should be noted that when $\phi$ equals to zero and one, the above equation is as same as the explicit Euler scheme and the implicit Euler scheme, respectively. In addition, the Crank-Nicolson scheme is derived when $\phi$ equals to $1 / 2$.

Now, adding $\phi^{2} A_{1} A_{2} V_{i, j}^{(n+1)}$ to both sides of the above equation

$$
\begin{aligned}
{\left[\mathbb{I}-\phi A_{0}-\right.} & \left.\phi A_{1}-\phi A_{2}+\phi^{2} A_{1} A_{2}\right] V_{i, j}^{(n+1)} \\
& =\left[\mathbb{I}+(1-\phi) A_{0}+(1-\phi) A_{1}+(1-\phi) A_{2}\right] V_{i, j}^{(n)}
\end{aligned}
$$




$$
\begin{gathered}
\quad+\phi^{2} A_{1} A_{2} V_{i, j}^{(n+1)}+\mathcal{O}\left((\Delta \tau)^{2}\right) \\
\Rightarrow \quad\left[\mathbb{I}-\phi A_{0}-\phi A_{1}-\phi A_{2}+\phi^{2} A_{1} A_{2}\right] V_{i, j}^{(n+1)} \\
=\left[\mathbb{I}+(1-\phi) A_{0}+(1-\phi) A_{1}+(1-\phi) A_{2}+\phi^{2} A_{1} A_{2}\right] V_{i, j}^{(n)} \\
+\phi^{2} A_{1} A_{2}\left(V_{i, j}^{(n+1)}-V_{i, j}^{(n)}\right)+\mathcal{O}\left((\Delta \tau)^{2}\right) \\
\Rightarrow \quad\left[\mathbb{I}-\phi A_{0}-\phi A_{1}-\phi A_{2}+\phi^{2} A_{1} A_{2}\right] V_{i, j}^{(n+1)} \\
=\left[\mathbb{I}+(1-\phi) A_{0}+(1-\phi) A_{1}+(1-\phi) A_{2}+\phi^{2} A_{1} A_{2}\right] V_{i, j}^{(n)}+\mathcal{O}\left((\Delta \tau)^{2}\right) \\
\Rightarrow \quad\left[\mathbb{I}-\phi A_{1}-\phi A_{2}+\phi^{2} A_{1} A_{2}\right] V_{i, j}^{(n+1)} \\
=\left[\mathbb{I}+A_{0}+(1-\phi) A_{1}+(1-\phi) A_{2}+\phi^{2} A_{1} A_{2}\right] V_{i, j}^{(n)} \\
\quad+\phi A_{0}\left(V_{i, j}^{(n+1)}-V_{i, j}^{(n)}\right)+\mathcal{O}\left((\Delta \tau)^{2}\right) \\
\Rightarrow \quad\left[\mathbb{I}-\phi A_{1}-\phi A_{2}+\phi^{2} A_{1} A_{2}\right] V_{i, j}^{(n+1)} \\
=\left[\mathbb{I}+A_{0}+(1-\phi) A_{1}+(1-\phi) A_{2}+\phi^{2} A_{1} A_{2}\right] V_{i, j}^{(n)}+\mathcal{O}\left((\Delta \tau)^{2}\right) \\
\Rightarrow \quad\left(\mathbb{I}-\phi A_{1}\right)\left(\mathbb{I}-\phi A_{2}\right) V_{i, j}^{(n+1)} \\
=\left[\mathbb{I}+A_{0}+(1-\phi) A_{1}+A_{2}\right] V_{i, j}^{(n)}-\left(\mathbb{I}-\phi A_{1}\right) \phi A_{2} V_{i, j}^{(n)},
\end{gathered}
$$

where two mergers appear since $\phi^{2} A_{1} A_{2}\left(V_{i, j}^{(n+1)}-V_{i, j}^{(n)}\right) \sim \mathcal{O}\left((\Delta \tau)^{3}\right)$ and $\phi A_{0}\left(V_{i, j}^{(n+1)}-\right.$ $\left.V_{i, j}^{(n)}\right) \sim \mathcal{O}\left((\Delta \tau)^{2}\right)$

In summary, the linear operators $A_{0}, A_{1}$ and $A_{2}$ at the $(n+1)$ th time step are

$$
\begin{aligned}
A_{0}= & \Delta \tau \cdot c_{j} \delta_{x v} \\
= & \Delta \tau \cdot\left[\rho \sigma v_{j}-\sigma^{2} v_{j} \xi_{j}\right] \delta_{x v} \\
A_{1}= & \Delta \tau\left[a_{j} \delta_{x x}+\left(d_{j}+\lambda_{j}\right) \delta_{x}-\frac{r}{2} \mathbb{I}\right] \\
= & \Delta \tau\left[\left(\frac{1}{2} v_{j}+\frac{1}{2} \sigma^{2} v_{j} \xi_{j}^{2}-\rho \sigma v_{j} \xi_{j}\right) \delta_{x x}\right. \\
& \left.+\left(-\frac{1}{2} v_{j}+\frac{1}{2} \sigma^{2} v_{j} \xi_{j}-\frac{1}{2} \sigma^{2} v_{j} \beta_{j}+r-D_{0}-\kappa\left(\eta-v_{j}\right) \xi_{j}+\lambda_{j}\right) \delta_{x}-\frac{r}{2} \mathbb{I}\right], \\
A_{2}= & \Delta \tau\left[b_{j} \delta_{v v}+e_{j} \delta_{v}-\frac{r}{2} \mathbb{I}\right] \\
= & \Delta \tau\left[\frac{1}{2} \sigma^{2} v_{j} \delta_{v v}+\kappa\left(\eta-v_{j}\right) \delta_{v}-\frac{r}{2} \mathbb{I}\right],
\end{aligned}
$$


where

$$
\begin{aligned}
& \xi_{j}=\frac{\delta_{v}\left(\phi S_{f}^{(n+1)}(j)+(1-\phi) S_{f}^{(n)}(j)\right)}{\phi S_{f}^{(n+1)}(j)+(1-\phi) S_{f}^{(n)}(j)}, \\
& \beta_{j}=\frac{\delta_{v v}\left(\phi S_{f}^{(n+1)}(j)+(1-\phi) S_{f}^{(n)}(j)\right)}{\phi S_{f}^{(n+1)}(j)+(1-\phi) S_{f}^{(n)}(j)}, \\
& \lambda_{j}=\frac{S_{f}^{(n+1)}(j)-S_{f}^{(n)}(j)}{\left(\phi S_{f}^{(n+1)}(j)+(1-\phi) S_{f}^{(n)}(j)\right) \Delta \tau} .
\end{aligned}
$$

\section{Appendix B}

$$
\mathbb{A} Y_{j}=P_{j}+\mathbb{B} x_{j},
$$

with

$$
\begin{aligned}
& \mathbb{A}=\left(\begin{array}{cccc}
1+\phi\left(\frac{2 a_{j} \Delta \tau}{(\Delta x)^{2}}+\frac{r \Delta \tau}{2}\right) & -\phi\left(\frac{a_{j} \Delta \tau}{(\Delta x)^{2}}+\frac{d_{j}^{\prime} \Delta \tau}{2 \Delta x}\right) & & \\
-\phi\left(\frac{a_{j} \Delta \tau}{(\Delta x)^{2}}-\frac{d_{j}^{\prime} \Delta \tau}{2 \Delta x}\right) & 1+\phi\left(\frac{2 a_{j} \Delta \tau}{(\Delta x)^{2}}+\frac{r \Delta \tau}{2}\right) & -\phi\left(\frac{a_{j} \Delta \tau}{(\Delta x)^{2}}+\frac{d_{j}^{\prime} \Delta \tau}{2 \Delta x}\right) & \\
\ddots & \ddots & \ddots & \\
& -\phi\left(\frac{a_{j} \Delta \tau}{(\Delta x)^{2}}-\frac{d_{j}^{\prime} \Delta \tau}{2 \Delta x}\right) & 1+\phi\left(\frac{2 a_{j} \Delta \tau}{(\Delta x)^{2}}+\frac{r \Delta \tau}{2}\right) & -\phi\left(\frac{a_{j} \Delta \tau}{(\Delta x)^{2}}+\frac{d_{j}^{\prime} \Delta \tau}{2 \Delta x}\right) \\
& & -\phi\left(\frac{a_{j} \Delta \tau}{(\Delta x)^{2}}-\frac{d_{j}^{\prime} \Delta \tau}{2 \Delta x}\right) & 1+\phi\left(\frac{2 a_{j} \Delta \tau}{(\Delta x)^{2}}+\frac{r \Delta \tau}{2}\right)
\end{array}\right)_{N_{x}-1 \times N_{x}-1} \\
& Y_{j}=\left(Y_{1, j}, Y_{2, j}, \cdots, Y_{N_{x}-1, j}\right)^{T}, \\
& P_{j}=\left(P_{1, j}, P_{1, j}, \cdots, P_{N_{x}-1, j}\right)^{T} \text {, } \\
& \mathbb{B} x_{j}=\left(\begin{array}{c}
\phi\left(\frac{a_{j} \Delta \tau}{(\Delta x)^{2}}-\frac{d_{j}^{\prime} \Delta \tau}{2 \Delta x}\right) Y_{0, j} \\
\vdots \\
\phi\left(\frac{a_{j} \Delta \tau}{(\Delta x)^{2}}+\frac{d_{j}^{\prime} \Delta \tau}{2 \Delta x}\right) Y_{N_{x}, j}
\end{array}\right)_{N_{x}-1 \times 1}, \\
& P_{i, j}=\left[\mathbb{I}+A_{0}+(1-\phi) A_{1}+A_{2}\right] V_{i, j}^{(n)} \\
& =V_{i, j}^{(n)}+\frac{c_{j} \Delta \tau}{4 \Delta x \Delta v}\left(V_{i+1, j+1}^{(n)}-V_{i+1, j-1}^{(n)}-V_{i-1, j+1}^{(n)}+V_{i-1, j-1}^{(n)}\right) \\
& +(1+\phi)\left(\frac{a_{j} \Delta \tau}{(\Delta x)^{2}}-\frac{d_{j}^{\prime} \Delta \tau}{2 \Delta x}\right) V_{i-1, j}^{(n)}+(1-\phi)\left(-\frac{a_{j} \Delta \tau}{(\Delta x)^{2}}-\frac{r \Delta \tau}{2}\right) V_{i, j}^{(n)} \\
& +(1-\phi)\left(\frac{a_{j} \Delta \tau}{(\Delta x)^{2}}+\frac{d_{j}^{\prime} \Delta \tau}{2 \Delta x}\right) V_{i+1, j}^{(n)}+\left(\frac{b_{j} \Delta \tau}{(\Delta v)^{2}}-\frac{e_{j} \Delta \tau}{2 \Delta v}\right) V_{i, j-1}^{(n)}
\end{aligned}
$$




$$
+\left(-\frac{b_{j} \Delta \tau}{(\Delta v)^{2}}-\frac{r \Delta \tau}{2}\right) V_{i, j}^{(n)}+\left(\frac{b_{j} \Delta \tau}{(\Delta v)^{2}}+\frac{e_{j} \Delta \tau}{2 \Delta v}\right) V_{i, j+1}^{(n)}
$$

where $d_{j}^{\prime}=d_{j}+\lambda_{j}$.

\section{Appendix $\mathrm{C}$}

$$
\mathbb{C} V_{i}^{(n+1)}=Q_{i}+\mathbb{B} v_{i}
$$

with

$$
\begin{aligned}
& \mathbb{C}=\left(\begin{array}{cccc}
1+\phi\left(\frac{2 b_{j} \Delta \tau}{(\Delta v)^{2}}+\frac{r \Delta \tau}{2}\right) & -\phi\left(\frac{b_{j} \Delta \tau}{(\Delta v)^{2}}+\frac{e_{j} \Delta \tau}{2 \Delta v}\right) & & \\
-\phi\left(\frac{b_{j} \Delta}{(\Delta v)^{2}}-\frac{e_{j} \Delta \tau}{2 \Delta v}\right) & 1+\phi\left(\frac{2 b_{j} \Delta \tau}{(\Delta v)^{2}}+\frac{r \Delta \tau}{2}\right) & -\phi\left(\frac{b_{j} \Delta \tau}{(\Delta v)^{2}}+\frac{e_{j} \Delta \tau}{2 \Delta v}\right) & \\
\ddots & \ddots & \ddots & \\
& -\phi\left(\frac{b_{j} \Delta}{(\Delta v)^{2}}-\frac{e_{j} \Delta \tau}{2 \Delta v}\right) & 1+\phi\left(\frac{2 b_{j} \Delta \tau}{(\Delta v)^{2}}+\frac{r \Delta \tau}{2}\right) & -\phi\left(\frac{b_{j} \Delta \tau}{(\Delta v)^{2}}+\frac{e_{j} \Delta \tau}{2 \Delta v}\right) \\
& & -\phi\left(\frac{b_{j} \Delta}{(\Delta v)^{2}}-\frac{e_{j} \Delta \tau}{2 \Delta v}\right) & 1+\phi\left(\frac{2 b_{j} \Delta \tau}{(\Delta v)^{2}}+\frac{r \Delta \tau}{2}\right)
\end{array}\right)_{N_{v} \times N_{v}} \\
& V_{i}^{(n+1)}=\left(V_{i, 1}^{(n+1)}, V_{i, 2}^{(n+1)}, \cdots, V_{i, N_{v}-1}^{(n+1)}\right)^{T}, \\
& Q_{i}^{(n+1)}=\left(q_{i, 1}, q_{i, 2}, \cdots, q_{i, N_{v}-1}\right)^{T}, \\
& \mathbb{B} v_{i}=\left(\begin{array}{c}
\phi\left(\frac{b_{j} \Delta \tau}{\Delta v}-\frac{e_{j} \Delta \tau}{2 \Delta v}\right) V_{i, 0}^{(n+1)} \\
\vdots \\
\phi\left(\frac{b_{j} \Delta \tau}{(\Delta v)^{2}}+\frac{e_{j} \Delta \tau}{2 \Delta v}\right) V_{i, N_{v}}^{(n+1)}
\end{array}\right)_{N_{v} \times 1}, \\
& q_{i, j}=Y_{i, j}-\phi\left(\frac{b_{j} \Delta \tau}{(\Delta v)^{2}}-\frac{e_{j} \Delta \tau}{2 \Delta v}\right) V_{i, j-1}^{(n)} \\
& -\phi\left(-2 \frac{b_{j} \Delta \tau}{(\Delta v)^{2}}-\frac{r \Delta \tau}{2}\right) V_{i, j}^{(n)}-\phi\left(\frac{b_{j} \Delta \tau}{(\Delta v)^{2}}+\frac{e_{j} \Delta \tau}{2 \Delta v}\right) V_{i, j+1}^{(n)} .
\end{aligned}
$$

\section{Appendix D}

$$
\begin{aligned}
A_{0} & =\Delta \tau \cdot c_{j} \delta_{x r} \\
& =\Delta \tau \cdot\left[\sigma \rho \xi \sqrt{r_{j}}-\xi^{2} r_{j} \zeta_{j}\right] \delta_{x r}
\end{aligned}
$$




$$
\begin{aligned}
A_{1}= & \Delta \tau\left[a_{j} \delta_{x x}+\left(d_{j}+\lambda_{j}\right) \delta_{x}-\frac{r_{j}}{2} \mathbb{I}\right] \\
= & \Delta \tau\left[\left(\frac{1}{2} \sigma^{2}+\frac{1}{2} \xi^{2} r_{j} \zeta_{j}^{2}-\sigma \rho \xi \sqrt{r_{j}} \zeta_{j}\right) \delta_{x x}\right. \\
& \left.+\left(-\frac{1}{2} \sigma^{2}+\frac{1}{2} \xi^{2} r_{j} \zeta_{j}^{2}-\frac{1}{2} \xi^{2} r_{j} \beta+r_{j}-D_{0}-\kappa\left(\eta-r_{j}\right) \zeta_{j}+\lambda_{j}\right) \delta_{x}-\frac{r_{j}}{2} \mathbb{I}\right], \\
A_{2}= & \Delta \tau\left[b_{j} \delta_{r r}+e_{j} \delta_{r}-\frac{r_{j}}{2} \mathbb{I}\right] \\
= & \Delta \tau\left[\frac{1}{2} \xi^{2} r_{j} \delta_{r r}+\kappa\left(\eta-r_{j}\right) \delta_{v}-\frac{r_{j}}{2} \mathbb{I}\right],
\end{aligned}
$$

where

$$
\begin{aligned}
& \zeta_{j}=\frac{\delta_{r}\left(\phi S_{c}^{(n+1)}(j)+(1-\phi) S_{c}^{(n)}(j)\right)}{\phi S_{c}^{(n+1)}(j)+(1-\phi) S_{c}^{(n)}(j)}, \\
& \beta_{j}=\frac{\delta_{r r}\left(\phi S_{c}^{(n+1)}(j)+(1-\phi) S_{c}^{(n)}(j)\right)}{\phi S_{c}^{(n+1)}(j)+(1-\phi) S_{c}^{(n)}(j)}, \\
& \lambda_{j}=\frac{S_{c}^{(n+1)}(j)-S_{c}^{(n)}(j)}{\left(\phi S_{c}^{(n+1)}(j)+(1-\phi) S_{c}^{(n)}(j)\right) \Delta \tau} .
\end{aligned}
$$

\section{Appendix E}

$$
\mathbb{A} Y_{j}=P_{j}+\mathbb{B} x_{j},
$$

with

$$
\begin{aligned}
& \mathbb{A}=\left(\begin{array}{cccc}
1+\phi\left(\frac{2 a_{j} \Delta \tau}{(\Delta x)^{2}}+\frac{r \Delta \tau}{2}\right) & -\phi\left(\frac{a_{j} \Delta \tau}{(\Delta x)^{2}}+\frac{d_{j}^{\prime} \Delta \tau}{2 \Delta x}\right) & & \\
-\phi\left(\frac{a_{j} \Delta \tau}{(\Delta x)^{2}}-\frac{d_{j}^{\prime} \Delta \tau}{2 \Delta x}\right) & 1+\phi\left(\frac{2 a_{j} \Delta \tau}{(\Delta x)^{2}}+\frac{r \Delta \tau}{2}\right) & -\phi\left(\frac{a_{j} \Delta \tau}{(\Delta x)^{2}}+\frac{d_{j}^{\prime} \Delta \tau}{2 \Delta x}\right) & \\
\ddots & \ddots & \ddots & \\
& -\phi\left(\frac{a_{j} \Delta \tau}{(\Delta x)^{2}}-\frac{d_{j}^{\prime} \Delta \tau}{2 \Delta x}\right) & 1+\phi\left(\frac{2 a_{j} \Delta \tau}{(\Delta x)^{2}}+\frac{r \Delta \tau}{2}\right) & -\phi\left(\frac{a_{j} \Delta \tau}{(\Delta x)^{2}}+\frac{d_{j}^{\prime} \Delta \tau}{2 \Delta x}\right) \\
& & -\phi\left(\frac{a_{j} \Delta \tau}{(\Delta x)^{2}}-\frac{d_{j}^{\prime} \Delta \tau}{2 \Delta x}\right) & 1+\phi\left(\frac{2 a_{j} \Delta \tau}{(\Delta x)^{2}}+\frac{r \Delta \tau}{2}\right)
\end{array}\right)_{N_{x}-1 \times N_{x}-1} \\
& Y_{j}=\left(Y_{1, j}, Y_{2, j}, \cdots, Y_{N_{x}-1, j}\right)^{T}, \\
& P_{j}=\left(P_{1, j}, P_{1, j}, \cdots, P_{N_{x}-1, j}\right)^{T},
\end{aligned}
$$




$$
\begin{aligned}
\mathbb{B} x_{j}= & \left(\begin{array}{c}
\phi\left(\frac{a_{j} \Delta \tau}{(\Delta x)^{2}}-\frac{d_{j}^{\prime} \Delta \tau}{2 \Delta x}\right) Y_{0, j} \\
\vdots \\
\phi\left(\frac{a_{j} \Delta \tau}{(\Delta x)^{2}}+\frac{d_{j}^{\prime} \Delta \tau}{2 \Delta x}\right) Y_{N_{x}, j}
\end{array}\right)_{N_{x}-1 \times 1}^{(n)} \\
P_{i, j}=\left[\mathbb{I}+A_{0}+(1-\phi) A_{1}+A_{2}\right] V_{i, j}^{(n)} & \\
=V_{i, j}^{(n)}+ & \frac{c_{j} \Delta \tau}{4 \Delta x \Delta r}\left(V_{i+1, j+1}^{(n)}-V_{i+1, j-1}^{(n)}-V_{i-1, j+1}^{(n)}+V_{i-1, j-1}^{(n)}\right) \\
& +(1+\phi)\left(\frac{a_{j} \Delta \tau}{(\Delta x)^{2}}-\frac{d_{j}^{\prime} \Delta \tau}{2 \Delta x}\right) V_{i-1, j}^{(n)}+(1-\phi)\left(-\frac{a_{j} \Delta \tau}{(\Delta x)^{2}}-\frac{r \Delta \tau}{2}\right) V_{i, j}^{(n)} \\
& +(1-\phi)\left(\frac{a_{j} \Delta \tau}{(\Delta x)^{2}}+\frac{d_{j}^{\prime} \Delta \tau}{2 \Delta x}\right) V_{i+1, j}^{(n)}+\left(\frac{b_{j} \Delta \tau}{(\Delta r)^{2}}-\frac{e_{j} \Delta \tau}{2 \Delta r}\right) V_{i, j-1}^{(n)} \\
& +\left(-\frac{b_{j} \Delta \tau}{(\Delta r)^{2}}-\frac{r \Delta \tau}{2}\right) V_{i, j}^{(n)}+\left(\frac{b_{j} \Delta \tau}{(\Delta r)^{2}}+\frac{e_{j} \Delta \tau}{2 \Delta r}\right) V_{i, j+1}^{(n)},
\end{aligned}
$$

where $d_{j}^{\prime}=d_{j}+\lambda_{j}$.

\section{Appendix F}

$$
\mathbb{C} V_{i}^{(n+1)}=Q_{i}+\mathbb{B} r_{i}
$$

with

$$
\begin{aligned}
& \mathbb{C}=\left(\begin{array}{cccc}
1+\phi\left(\frac{2 b_{j} \Delta \tau}{(\Delta r)^{2}}+\frac{r \Delta \tau}{2}\right) & -\phi\left(\frac{b_{j} \Delta \tau}{(\Delta r)^{2}}+\frac{e_{j} \Delta \tau}{2 \Delta r}\right) & & \\
-\phi\left(\frac{b_{j} \Delta}{(\Delta r)^{2}}-\frac{e_{j} \Delta \tau}{2 \Delta r}\right) & 1+\phi\left(\frac{2 b_{j} \Delta \tau}{(\Delta r)^{2}}+\frac{r \Delta \tau}{2}\right) & -\phi\left(\frac{b_{j} \Delta \tau}{(\Delta r)^{2}}+\frac{e_{j} \Delta \tau}{2 \Delta r}\right) & \\
\ddots & \ddots & \ddots & \\
& -\phi\left(\frac{b_{j} \Delta}{(\Delta r)^{2}}-\frac{e_{j} \Delta \tau}{2 \Delta r}\right) & 1+\phi\left(\frac{2 b_{j} \Delta \tau}{(\Delta r)^{2}}+\frac{r \Delta \tau}{2}\right) & -\phi\left(\frac{b_{j} \Delta \tau}{(\Delta r)^{2}}+\frac{e_{j} \Delta \tau}{2 \Delta r}\right) \\
& & -\phi\left(\frac{b_{j} \Delta}{(\Delta r)^{2}}-\frac{e_{j} \Delta \tau}{2 \Delta r}\right) & 1+\phi\left(\frac{2 b_{j} \Delta \tau}{(\Delta r)^{2}}+\frac{r \Delta \tau}{2}\right)
\end{array}\right)_{N_{r} \times N_{r}} \\
& V_{i}^{(n+1)}=\left(V_{i, 1}^{(n+1)}, V_{i, 2}^{(n+1)}, \cdots, V_{i, N_{r}-1}^{(n+1)}\right)^{T}, \\
& Q_{i}^{(n+1)}=\left(q_{i, 1}, q_{i, 2}, \cdots, q_{i, N_{r}-1}\right)^{T}, \\
& \mathbb{B} v_{i}=\left(\begin{array}{c}
\phi\left(\frac{b_{j} \Delta \tau}{\Delta r}-\frac{e_{j} \Delta \tau}{2 \Delta r}\right) V_{i, 0}^{(n+1)} \\
\vdots \\
\phi\left(\frac{b_{j} \Delta \tau}{(\Delta r)^{2}}+\frac{e_{j} \Delta \tau}{2 \Delta r}\right) V_{i, N_{r}}^{(n+1)}
\end{array}\right)_{N_{r} \times 1},
\end{aligned}
$$




$$
\begin{aligned}
q_{i, j}=Y_{i, j} & -\phi\left(\frac{b_{j} \Delta \tau}{(\Delta r)^{2}}-\frac{e_{j} \Delta \tau}{2 \Delta r}\right) V_{i, j-1}^{(n)} \\
& -\phi\left(-2 \frac{b_{j} \Delta \tau}{(\Delta r)^{2}}-\frac{r \Delta \tau}{2}\right) V_{i, j}^{(n)}-\phi\left(\frac{b_{j} \Delta \tau}{(\Delta r)^{2}}+\frac{e_{j} \Delta \tau}{2 \Delta r}\right) V_{i, j+1}^{(n)}
\end{aligned}
$$

\section{References}

[1] M. Ammann, A. Kind, and C. Wilde. Simulation-based pricing of convertible bonds. Journal of Empirical Finance, 15(2):310-331, 2008.

[2] G. Barone-Adesi, A. Bermúdez, and J. Hatgioannides. Two-factor convertible bonds valuation using the method of characteristics/finite elements. Journal of Economic Dynamics and Control, 27(10):1801-1831, 2003.

[3] F. Black and M. Scholes. The pricing of options and corporate liabilities. Journal of political economy, 81(3):637-654, 1973.

[4] M. J. Brennan and E. S. Schwartz. Convertible bonds: Valuation and optimal strategies for call and conversion. The Journal of Finance, 32(5):1699-1715, 1977.

[5] M. J. Brennan and E. S. Schwartz. Analyzing convertible bonds. Journal of Financial and Quantitative analysis, 15(04):907-929, 1980.

[6] J. C. Butcher. Numerical methods for ordinary differential equations. John Wiley \& Sons, 2016.

[7] D. R. Chambers and Q. Lu. A tree model for pricing convertible bonds with equity, interest rate, and default risk. The Journal of Derivatives, 14(4):25-46, 2007.

[8] W. Chen, L. Xu, and S.-P. Zhu. Stock loan valuation under a stochastic interest rate model. Computers \& Mathematics with Applications, 70(8):1757-1771, 2015.

[9] J. Cox. Notes on option pricing i: Constant elasticity of variance diffusions. Unpublished note, Stanford University, Graduate School of Business, 1975. 
[10] J. C. Cox, J. E. Ingersoll, and S. A. Ross. An analysis of variable rate loan contracts. The Journal of Finance, 35(2):389-403, 1980.

[11] J. C. Cox, J. E. Ingersoll Jr, and S. A. Ross. A theory of the term structure of interest rates. Econometrica: Journal of the Econometric Society, pages 385-407, 1985.

[12] J. C. Cox and S. A. Ross. The valuation of options for alternative stochastic processes. Journal of financial economics, 3(1-2):145-166, 1976.

[13] I. J. Craig and A. D. Sneyd. An alternating-direction implicit scheme for parabolic equations with mixed derivatives. Computers \&6 Mathematics with Applications, 16(4):341-350, 1988.

[14] W. Dai and R. Nassar. A second-order ADI scheme for three-dimensional parabolic differential equations. Numerical Methods for Partial Differential Equations, 14(2):159$168,1998$.

[15] L. U. Dothan. On the term structure of interest rates. Journal of Financial Economics, 6(1):59-69, 1978.

[16] E. Ekström, P. Lötstedt, and J. Tysk. Boundary values and finite difference methods for the single factor term structure equation. Applied Mathematical Finance, 16(3):253-259, 2009.

[17] G. Fichera. On a unified theory of boundary value problems for elliptic-parabolic equations of second order. Mathematics Research Center, United States Army, University of Wisconsin, 1959.

[18] T. Haentjens and K. J. Hout. ADI finite difference schemes for the Heston-Hull-White PDE. arXiv preprint arXiv:1111.4087, 2011.

[19] X.-J. He and S.-P. Zhu. A closed-form pricing formula for European options under 
the Heston model with stochastic interest rate. Journal of Computational and Applied Mathematics, 335:323-333, 2018.

[20] S. L. Heston. A closed-form solution for options with stochastic volatility with applications to bond and currency options. Review of financial studies, 6(2):327-343, 1993.

[21] J. Hull and A. White. The pricing of options on assets with stochastic volatilities. The journal of finance, 42(2):281-300, 1987.

[22] W. Hundsdorfer. Accuracy and stability of splitting with stabilizing corrections. Applied Numerical Mathematics, 42(1-3):213-233, 2002.

[23] M.-W. Hung and J.-Y. Wang. Pricing convertible bonds subject to default risk. The journal of Derivatives, 10(2):75-87, 2002.

[24] S. Ikonen and J. Toivanen. Efficient numerical methods for pricing american options under stochastic volatility. Numerical Methods for Partial Differential Equations, 24(1):104-126, 2008.

[25] J. E. Ingersoll. A contingent-claims valuation of convertible securities. Journal of Financial Economics, 4(3):289-321, 1977.

[26] T. Kimura and T. Shinohara. Monte Carlo analysis of convertible bonds with reset clauses. European Journal of Operational Research, 168(2):301-310, 2006.

[27] H. G. Landau. Heat conduction in a melting solid. Quarterly of Applied Mathematics, $8(1): 81-94,1950$.

[28] D. Lvov, A. B. Yigitbasioglu, and N. El Bachir. Pricing convertible bonds by simulation. In Second IASTED international conference, pages 259-264, 2004.

[29] T. A. Marsh and E. R. Rosenfeld. Stochastic processes for interest rates and equilibrium bond prices. The Journal of Finance, 38(2):635-646, 1983. 
[30] J. McConnell and E. S. Schwartz. LYON taming. The Journal of Finance, 41(3):561$576,1986$.

[31] R. C. Merton. Theory of rational option pricing. The Bell Journal of economics and management science, pages 141-183, 1973.

[32] K. G. Nyborg. The use and pricing of convertible bonds. Applied Mathematical Finance, 3(3):167-190, 1996.

[33] F. Ohtake, N. Oda, T. Yoshiba, et al. Market price analysis and risk management for convertible bonds. Monetary and Economic Studies, 17(2):47-89, 1999.

[34] D. W. Peaceman and H. H. Rachford, Jr. The numerical solution of parabolic and elliptic differential equations. Journal of the Society for industrial and Applied Mathematics, 3(1):28-41, 1955.

[35] W. H. Press. Numerical recipes 3rd edition: The art of scientific computing. Cambridge university press, 2007.

[36] J. C. Strikwerda. Finite difference schemes and partial differential equations. 1989.

[37] J. C. Strikwerda. Finite difference schemes and partial differential equations, volume 88. Siam, 2004.

[38] K. Tsiveriotis and C. Fernandes. Valuing convertible bonds. Journal of Fixed Income, 8(2):95-102, 1998.

[39] O. Vasicek. An equilibrium characterization of the term structure. Journal of financial economics, 5(2):177-188, 1977.

[40] P. Wilmott, J. Dewynne, and S. Howison. Option pricing: Mathematical models and computation. Oxford financial press, 1993. 
[41] Z. Yang and F. Yi. A free boundary problem arising from pricing convertible bond. Applicable Analysis, 89(3):307-323, 2010.

[42] A. B. Yigitbasioglu. Pricing convertible bonds with interest rate, equity, credit, and fx risk. 2002.

[43] S.-P. Zhu. A closed-form analytical solution for the valuation of convertible bonds with constant dividend yield. The ANZIAM Journal, 47(04):477-494, 2006.

[44] S.-P. Zhu and W.-T. Chen. A predictor-corrector scheme based on the ADI method for pricing American puts with stochastic volatility. Computers 83 Mathematics with Applications, 62(1):1-26, 2011.

[45] S.-P. Zhu, S. Lin, and X. Lu. Pricing puttable convertible bonds with integral equation approaches. Computers $\& 3$ Mathematics with Applications, 75(8):2757-2781, 2018.

[46] S.-P. Zhu and J. Zhang. A new predictor-corrector scheme for valuing American puts. Applied Mathematics and Computation, 217(9):4439-4452, 2011.

[47] R. Zvan, P. A. Forsyth, and K. Vetzal. A finite volume approach for contingent claims valuation. IMA Journal of Numerical Analysis, 21(3):703-731, 2001. 